\title{
TOEPLITZ OPERATORS AND WEIGHTED WIENER-HOPF OPERATORS, PSEUDOCONVEX REINHARDT AND TUBE DOMAINS
}

\author{
NORBERTO SALINAS
}

\begin{abstract}
The notion of weighted Wiener-Hopf operators is introduced. Their relationship with Toeplitz operators acting on the space of holomorphic functions which are square integrable with respect to a given "symmetric" measure is discussed. The groupoid approach is used in order to present a general program for studying the $C^{*}$-algebra generated by weighted Wiener-Hopf operators associated with a solid cone of a second countable locally compact Hausdorff group. This is applied to the case when the group is the dual of a connected locally compact abelian Lie group and the measure is "well behaved" in order to produce a geometric groupoid which is independent of the representation. The notion of a Reinhardt-tube domain $\Omega$ appears thus naturally, and a decomposition series of the corresponding $C^{*}$-algebra is presented in terms of groupoid $C^{*}$-algebras associated with various parts of the boundary of the domain $\Omega$.
\end{abstract}

\section{INTRODUCTION}

Our purpose in this note is to call attention to an important connection between Toeplitz operators acting on the subspace of square integrable holomorphic functions with respect to a symmetric measure supported on a (symmetric) domain $\Omega \subseteq \mathbf{C}^{n}$ and weighted Wiener-Hopf operators on the dual set $\widehat{\Omega}$. In the present paper we consider, in particular, two types of symmetric domains: (a) Reinhardt domains (which are domains invariant under the action of the $n$-torus $\mathbf{T}^{n}$ by coordinatewise multiplication), and (b) tube domains (which are domains invariant under the $\mathbf{R}^{n}$ translations in the imaginary direction). We also consider (c) Reinhardt-tube domains (which are domains of mixed type).

The above relationship between Toeplitz operators and weighted WienerHopf operators is specially relevant when studying the structure of the $C^{*}$ algebras generated by such operators. More general symmetries are considered in [20] based on generalized Reinhardt domains [1], where analogous structure properties of the corresponding $C^{*}$-algebras are studied.

We also present a general program exploiting a useful technique (already present in the literature for some particular cases, see $[11,4,19]$ ) based on groupoids, to investigate the properties of the $C^{*}$-algebra generated by weighted Wiener-Hopf operators on certain semigroups (that we call cones) of a locally compact second countable Hausdorff group. We construct a groupoid $\mathscr{G}$ so that

Received by the editors December 19, 1990.

1980 Mathematics Subject Classification (1985 Revision). Primary 46L05.

This paper was supported in part by a grant of the National Science Foundation. 
the Wiener-Hopf $C^{*}$-algebra associated with the cone $\Gamma$ and weight function $\omega$ is a natural subalgebra of $C_{\text {red }}^{*}(\mathscr{G})$.

The groupoid approach to study $C^{*}$-algebras was formally introduced in [16], and is very helpful in finding decomposition series of a $C^{*}$-algebra in terms of various reductions of the groupoid. Our final task is to suggest, under mild assumptions on the measure supported on the closure of the domain $\Omega$ (what we call well behaved measures), a technique that produces a groupoid $\mathscr{G}(\Omega)$ and that relates such reductions of $\mathscr{G}$ to $\partial \Omega$. In fact, we present a suitable parameterization of the groupoid $\mathscr{G}(\Omega)$ that takes into account the boundary geometry of the domain $\Omega$. Here is where pseudoconvexity of $\Omega$ enters successfully into the picture. This connection was already observed in [19] for the case in which the measure was the volume Lebesgue measure on a bounded complete Reinhardt domain. More recently, extensions of the results of [19] were announced in [3], for the case of well behaved radial measures supported on the same kind of domains.

The organization of the paper is as follows: in $\S 2$, we introduce some terminology and consider the case of pseudoconvex Reinhardt bounded (connected) domains $\Omega$. As an important typical example, we discuss the generalized Hartogs wedge. Then, under a natural assumption on the Reinhardt measure $\mu$ supported on a more general domain $\Omega$, we prove that the structure of the corresponding Toeplitz $C^{*}$-algebra over $\Omega$ depends only on the boundary geometry of $\Omega$, and we extend to arbitrary pseudoconvex Reinhardt domains $\Omega \subseteq \mathbf{C}^{2}$, results obtained in $[19,22]$. Some of these results are also extended to subsets of $\mathbf{C}^{n}$. In $\S 3$, we present the case of pseudoconvex tube domains $\Omega$. We conjecture that this corresponding Wiener-Hopf $C^{*}$-algebra is always type I. A positive answer to this conjecture would extend the result for unweighted Wiener-Hopf $C^{*}$-algebras on polyhedral cones obtained in [11]. Finally, in $\S 4$, we describe a general procedure for studying the $C^{*}$-algebra generated by weighted Wiener-Hopf operators on a cone $\Gamma$ of a second countable locally compact Hausdorff group $G$, where the weight function is a quasidecreasing strictly positive Borel function on $\Gamma$. We apply these techniques to the case in which $G$ is the dual of a connected locally compact abelian Lie group, i.e., a group of the form $\mathbf{Z}^{m} \times \mathbf{R}^{n}$ (see [6, Appendix B25]). Here is where pseudoconvex Reinhardt-tube domains enter in our discussion. With these types of domains, we unify the results obtained in $[4,11,19]$.

\section{Pseudoconvex Reinhardt domains}

Throughout this section, $\Omega$ will denote a bounded pseudoconvex Reinhardt (connected) domain. We recall that $\Omega$ is called a Reinhardt domain (see [7, $\S 2.4$, and 15]) when given any two $n$-tuples $z=\left(z_{1}, \ldots, z_{n}\right) \in \Omega$ and $t=$ $\left(t_{1}, \ldots, t_{n}\right) \in \mathbf{T}^{n}$, the $n$-tuple $\left(t_{1} z_{1}, \ldots, t_{n} z_{n}\right)$ is in $\Omega$. The domain $\Omega$ is pseudoconvex (or a domain of holomorphy) when it is logarithmically convex, i.e., when the domain

$$
\log \left(\Omega_{+}\right)=\left\{x=\left(x_{1}, \ldots, x_{n}\right) \in \mathbf{R}^{n}: \operatorname{Exp}(x)=\left(e^{x_{1}}, \ldots, e^{x_{n}}\right) \in \Omega\right\},
$$

is convex in $\mathbf{R}^{n}$, where $\Omega_{+}=\Omega \cap \mathbf{R}_{+}^{n}$. A Reinhardt domain $\Omega$ is called complete if given $z \in \Omega$, the whole polydisk with center at the origin and multiradius $\left(\left|z_{1}\right|, \ldots,\left|z_{n}\right|\right)$ is contained in $\Omega$. A pseudoconvex Reinhardt domain $\Omega$ contains the origin if and only if it is complete. We present below the 
example of the generalized Hartogs wedge which is a pseudoconvex Reinhardt domain that contains the origin in its boundary (and therefore is not complete).

We let $B^{2}(\Omega)$ denote the Bergman space (of holomorphic functions) in $L^{2}(\Omega)$ which is the standard Hilbert space of square-integrable functions on $\Omega$ with respect to volume Lebesgue measure $d V$.

The unital $C^{*}$-algebra $C^{*}\left(T_{Z}\right)$ generated by the $n$-tuple $T_{Z}$, where $Z=$ $\left(Z_{1}, \ldots, Z_{n}\right)$ is the $n$-tuple of coordinate functions $Z_{j}: \Omega \rightarrow \mathbf{C}, 1 \leq j \leq n$, acting on $B^{2}(\Omega)$ coincides with the $C^{*}$-algebra generated by Toeplitz operators $T_{\phi}$ with continuous symbol $\phi$ on $\bar{\Omega}$ (this is an immediate consequence of the Stone-Weierstrass theorem and the compactness of $\bar{\Omega}$ ).

One of the important features of Reinhardt domains is that the set

$$
\left\{Z^{\nu}=\left(Z_{1}^{\nu_{1}}, \ldots, Z_{n}^{\nu_{n}}\right) \in L^{2}(\Omega)\right\}
$$

constitutes an orthogonal basis for $B^{2}(\Omega)$. We denote by $B_{\Omega}$ by the set of multi-indices corresponding to the above basis 1 . Let

$$
\Gamma_{\Omega}=\left\{\mu \in \mathbf{Z}^{n}: Z^{\mu} \in H^{\infty}(\Omega)\right\} .
$$

It is obvious that $\Gamma_{\Omega} \subseteq B_{\Omega}$ and that $B_{\Omega}$ is an invariant subset of $\mathbf{Z}^{n}$ under translations by elements of $\Gamma_{\Omega}$.

From (2), it follows that, for each $\mu \in \Gamma_{\Omega}$, the Toeplitz operator $T_{Z^{\mu}}$ is bounded on $B^{2}(\Omega)$ and can be expressed as a weighted shift operator on the orthonormal basis given by

$$
E_{\nu}(z)=\frac{z^{\nu}}{\left\|Z^{\nu}\right\|}, \quad \nu \in B_{\Omega}, \quad z \in \Omega,
$$

with weight sequence

$$
w_{\mu}(\nu)=\left\|Z^{\mu+\nu}\right\| /\left\|Z^{\nu}\right\|, \quad \mu \in \Gamma_{\Omega}, \quad \nu \in B_{\Omega} .
$$

In studying the spectral and $C^{*}$-algebraic properties of $T_{Z}$, it is obviously enough to assume, after a suitable scaie dilation (a diagonal expansion and/or contraction), that $\Omega$ is contained in the unit polydisk $\mathbf{D}^{n}$, and that $\Omega_{+} \cap \partial \mathbf{D}_{+}^{n}$ is maximal. Geometrically, this condition means that the closure of the convex set $\log \left(\Omega_{+}\right)$touches (perhaps tangentially) all the coordinate hyperplanes of the negative orthant $\mathbf{R}_{-}^{n}$. We make this normalizing assumption throughout the rest of the present section. Observe that we then have $\mathbf{Z}_{+}^{n} \subseteq \Gamma_{\Omega}$. Moreover, there are certain geometrical properties related to $B_{\Omega}$ and $\Gamma_{\Omega}$ that will become apparent once we introduce the following terminology: let

$$
\begin{aligned}
& B_{\Omega}^{\prime}=\left\{\dot{\rho} \in \mathbf{R}^{n}: \prod_{j=1}^{n}\left|Z_{j}\right|^{\rho_{j}} \in L^{2}(\Omega)\right\}, \\
& \Gamma_{\Omega}^{\prime}=\left\{\sigma \in \mathbf{R}^{n}: \prod_{j=1}^{n}\left|Z_{j}\right|^{\sigma_{j}} \in L^{\infty}(\Omega)\right\} .
\end{aligned}
$$

Obviously, $B_{\Omega}=B_{\Omega}^{\prime} \cap \mathbf{Z}^{n}$, and $\Gamma_{\Omega}=\Gamma_{\Omega}^{\prime} \cap \mathbf{Z}^{n}$.

Lemma 2.1. The sets $B_{\Omega}^{\prime}$ and $\Gamma_{\Omega}^{\prime}$ are convex, and $\Gamma_{\Omega}^{\prime}$ is a cone whose closure coincides with $\overline{\operatorname{conv}\left(\Gamma_{\Omega}\right)}$. 
Proof. The fact that $\Gamma_{\Omega}^{\prime}$ is a convex cone whose closure is solid is an immediate consequence of the definition. Suppose that $\rho$ and $\sigma$ are in $B_{\Omega}^{\prime}$, and let $a>0$, $b>0$ be such that $a+b=1$. Then, by Holder's inequality, we have

$$
\begin{aligned}
\int_{\Omega}\left|z^{2 a \rho+2 b \sigma}\right| d V(z) & =(2 \pi)^{n} \int_{\Omega_{+}} r^{2 a \rho} r^{2 b \sigma} r^{n} d V(r) \\
& \leq(2 \pi)^{n}\left(\int_{\Omega_{+}} r^{2 \rho} r^{n} d V(r)\right)^{a}\left(\int_{\Omega_{+}} r^{2 \sigma} r^{n} d V(r)\right)^{b}<\infty .
\end{aligned}
$$

Given a set $A \subseteq \mathbf{R}^{n}$ such that the origin is not in the interior of the convex hull $\operatorname{conv}(A)$, let $\operatorname{con}(A)$ be the cone in $\mathbf{R}^{n}$ generated by $A$. Also, let $\hat{A}$ be the dual cone of $A$, i.e.,

$$
\widehat{A}=\left\{x \in \mathbf{R}^{n}:\langle x, y\rangle>0, \forall y \in A\right\} .
$$

Observe that $\hat{A}=\widehat{\operatorname{con}(A)}$. It is now easy to check that

$$
x \in \log \left(\Omega_{+}\right) \Leftrightarrow k x \in \log \left(\Omega_{+}\right), \quad k=1,2, \ldots,
$$

if and only if

$$
\overline{\Gamma_{\Omega}^{\prime}}=\overline{\overline{\left(\log \left(\Omega_{+}\right)\right.}} \text {. }
$$

Furthermore, condition $(*)$ is also equivalent to the following:

$\operatorname{con}\left(\log \left(\Omega_{+}\right)\right)$is a cone that contains no lines, and all the (half)rays

in the boundary of $\operatorname{con}\left(\log \left(\Omega_{+}\right)\right)$are tangent to $\log \left(\Omega_{+}\right)$at $\infty$.

Thus, condition $(*)$ is satisfied when $\Omega$ is a complete (normalized pseudoconvex) Reinhardt domain. In this case, $\left\{z \in \mathbf{C}^{n}: \prod_{j=1}^{n} z_{j}=0\right\} \subseteq \Omega$, and $\Gamma_{\Omega}=\mathbf{Z}_{+}^{n}$. On the other hand, a Reinhardt domain $\Omega$ such that $\log \left(\Omega_{+}\right)$is bounded does not satisfy $(*)$. In this case $\Omega \cap\left\{z \in \mathbf{C}^{n}: \prod_{j=1}^{n} z_{j}=0\right\}=\varnothing$ and $\Gamma_{\Omega}=\mathbf{Z}^{n}$.

Lemma 2.2. Let 1 denote the multi-index $1=(1, \ldots, 1)$, and assume that condition $(*)$ is satisfied. Then, $\mathbf{1}+\overline{B_{\Omega}^{\prime}} \subseteq \overline{\Gamma_{\Omega}^{\prime}}$.

Proof. Assume that $\rho \in B_{\Omega}^{\prime}$. Then

$$
\begin{aligned}
\left\|Z^{\rho}\right\|^{2} & =(2 \pi)^{n} \int_{\Omega_{+}} r^{2 \rho} r^{n} d V(r) \\
& =(2 \pi)^{n} \int_{\log \left(\Omega_{+}\right)} e^{2\langle\rho+1, x\rangle} d V(x)<\infty .
\end{aligned}
$$

If $\langle\rho+1, y\rangle>0$ for some $y \in \log \left(\Omega_{+}\right)$, then the same property holds for every vector in a (small) open cone $C$ symmetric around $y$. This implies that

$$
\int_{C \cap \log \left(\Omega_{+}\right)} e^{2\langle\rho+1, x\rangle} d V(x)=\infty,
$$

which contradicts $\rho \in B_{\Omega}^{\prime}$. We deduce that $\rho+1 \in \overline{\overline{\left(-\log \left(\Omega_{+}\right)\right)}}$. From condition $(*)$, we conclude that $\rho+1 \in \overline{\Gamma_{\Omega}^{\prime}}$, as desired.

We shall see below a simple example where the inclusion in the above lemma becomes an equality. Such an inclusion together with the obvious inclusion $\Gamma_{\Omega}^{\prime} \subseteq B_{\Omega}^{\prime}$ implies that $\Gamma_{\Omega}$ is the largest cone in $\mathbf{Z}^{n}$ for which $B_{\Omega}$ is an invariant set. 
Lemma 2.3. The weight function

$$
\begin{aligned}
& \omega(\nu)=\left\|Z^{\nu}\right\|, \quad \nu \in B_{\Omega}, \\
& \omega(\nu)=0, \quad \nu \in \mathbf{Z}^{n} \backslash B_{\Omega},
\end{aligned}
$$

is a nonnegative function whose support is $B_{\Omega}$ and satisfies

$$
\sup _{\nu \in B_{\Omega}} \frac{\omega(\mu+\nu)}{\omega(\nu)}=\zeta(\mu)<\infty, \quad \forall \mu \in \Gamma_{\Omega} .
$$

Furthermore, if $\Omega$ satisfies condition $(*)$, then $\zeta(\mu)=1, \forall \mu \in \Gamma_{\Omega}$ (in other words, $\omega$ is decreasing on $B_{\Omega}$ with respect to the partial order induced by $\Gamma_{\Omega}$ ). Proof. The first estimate is a consequence of the definitions of $\Gamma_{\Omega}$ (see (2)) and $B_{\Omega}$, where $\zeta(\mu)=\left\|Z^{\mu}\right\|_{\infty}, \mu \in \Gamma_{\Omega}$. Now, if $\Omega$ satisfies condition (*), it follows, from the proof of the previous lemma, that $\left\|Z^{\mu}\right\|_{\infty}=1, \mu \in \Gamma_{\Omega}$. This completes the proof of the last assertion.

Remark 2.4. (a) An immediate consequence of the above lemma is that, in the case that $\Omega$ satisfies condition $(*)$, the operators $T_{Z^{\nu}}, \nu \in \Gamma_{\Omega}$, are all contractions.

(b) In general, we have

$$
\left\|T_{Z^{\mu}}\right\|=\left\|Z^{\mu}\right\|_{\infty}=\zeta(\nu), \quad \mu \in \Gamma_{\Omega} .
$$

(See the proof of Theorem 2.17.) Let $l^{2}(\omega)$ be the Hilbert space of squaresummable sequences $\left\{g_{\nu}: \nu \in \mathbf{Z}^{n}\right\}$ with weight sequence $\omega$, i.e., $g \in l^{2}(\omega)$ if and only if

$$
\sum_{\nu \in \mathbf{Z}^{n}}\left|g_{\nu}\right|^{2} \omega^{2}(\nu)=\sum_{\nu \in B_{\Omega}}|g(\nu)|^{2} \omega^{2}(\nu)<\infty
$$

Likewise, let $l^{1}(\zeta)$ be the Banach space of summable sequences with weight sequence

$$
\begin{array}{ll}
\zeta(\mu)=\sup _{\nu \in B_{\Omega}} \frac{\omega(\mu+\nu)}{\omega(\nu)}, & \mu \in \Gamma_{\Omega}, \\
\zeta(\mu)=0, \quad \mu \notin \Gamma_{\Omega} . &
\end{array}
$$

It readily follows that $l^{1}(\zeta)$ is actually a Banach algebra under (standard) convolution. Indeed, first observe that $\zeta$ has the following submultiplicative property

$$
\zeta(\mu) \leq \zeta(\lambda) \zeta(\mu-\lambda), \quad \forall \mu, \lambda, \mu-\lambda \in \Gamma_{\Omega} .
$$

To prove this, we recall that

$$
\zeta(\mu)=\sup _{\nu-\mu \in B_{\Omega}} \frac{\omega(\nu)}{\omega(\nu-\mu)} .
$$

Therefore, for every $\lambda \in \Gamma_{\Omega}, \nu-\mu \in \Gamma_{\Omega}$, we have $\omega(\lambda+\nu-\mu) / \omega(\nu-\mu)$ $\leq \zeta(\lambda)$. Also, if $\nu-\mu \in B_{\Omega}, \mu, \lambda, \mu-\lambda \in \Gamma_{\Omega}$ (so that $\nu \in B_{\Omega}$ ), we have $\omega(\nu) / \omega(\lambda+\nu-\mu) \leq \zeta(\mu-\lambda)$. Thus, by dividing and multiplying by $\omega(\lambda+\nu-\mu), \mu, \lambda, \mu-\lambda \in \Gamma_{\Omega}, \nu-\mu \in B_{\Omega}$ on the right-hand side of (9), we obtain (8), as claimed. Now, let $\phi, \psi \in l^{1}(\zeta)$. Then

$$
\begin{aligned}
\|\phi * \psi\|_{1, \zeta} & =\sum_{\mu \in \Gamma_{\Omega}}|\phi * \psi(\mu)| \zeta(\mu) \\
& \leq \sum_{\mu \in \Gamma_{\Omega}} \sum_{\lambda \in \Gamma_{\Omega}}|\phi(\lambda)| \zeta(\lambda)|\psi(\mu-\lambda)| \theta(\mu-\lambda) \\
& \leq\|\phi\|_{1, \zeta}\|\psi\|_{1, \zeta} .
\end{aligned}
$$


(c) Following the ideas in [23], we can regard the operators $T_{Z^{\nu}}$ as either weighted shifts on a standard $l^{2}$-space, or unweighted shifts on a weighted $l^{2}$ space. This latter point of view is the most suitable to define more general Wiener-Hopf operators.

Given $\phi \in l^{1}(\zeta)$, (so that $\operatorname{supp}(\phi) \subseteq \Gamma_{\Omega}$ ) we define the Wiener-Hopf operator $W_{\phi}$, with symbol $\phi$, on $l^{2}(\omega)$, by

$$
\begin{gathered}
\left(W_{\phi} g\right)(\mu)=\phi * g(\nu)=\sum_{\nu} \phi(\mu) g(\nu-\mu), \\
g \in l^{2}(\omega), \quad \nu \in B_{\Omega} .
\end{gathered}
$$

In other words, $W_{\phi}$ is defined as the unweighted convolution on $l^{2}(\omega)$. (Note that $l^{2}(\omega)$ can be considered as a space of functions supported on $B_{\Omega}$.) It is straightforward to check that $W_{\phi}$ is a bounded operator. Indeed,

$$
\begin{aligned}
\left\|W_{\phi} g\right\|_{2, \omega}^{2} & =\sum_{\nu} \omega^{2}(\nu)\left|\sum_{\mu} \phi(\mu) g(\nu-\mu)\right|^{2} \\
& \leq \sum_{\nu}\left[\sum_{\mu}|\phi(\nu)| \zeta(\nu)|g(\mu-\nu)| \omega(\mu-\nu)\right]^{2} \\
& \leq\|\phi\|_{1, \zeta}^{2}\|g\|_{2, \omega}^{2} .
\end{aligned}
$$

If $\phi_{\mu}$ denotes the sequence which is the Dirac mass at $\mu \in \Gamma_{\Omega}$, then $\phi_{\mu} \in l^{1}(\zeta)$, and it is immediate that $W_{\phi_{\mu}}$ coincides with the unweighted forward shift $S_{\mu}$, defined on $l^{2}(\omega)$, by

$$
\left(S_{\mu} g\right)(\nu)=g(\nu-\mu), \quad \nu \in B_{\Omega} .
$$

In other words, $W_{\phi_{\mu}}$ is unitarily equivalent to $T_{Z^{\mu}}, \mu \in \Gamma_{\Omega}$. It is natural then to define the weighted Wiener-Hopf $C^{*}$-algebra $\mathscr{W}(\omega)$ as the $C^{*}$-algebra generated by $W_{\phi}, \phi \in l^{1}(\zeta)$. When the weight function $\omega$ is constant, i.e., the (unweighted) standard case, $\mathscr{W}(\omega)$ coincides with the usual definition (in this case $W_{\phi}$ can be defined for any $\phi$ in $\left.l^{1}\left(\mathbf{Z}^{n}\right)\right)$. This is the $C^{*}$-algebra considered in [13].

(d) If $\omega$ is computed using the norm in $B^{2}(\Omega)$ as above, then it follows from our previous discussion, $\mathscr{W}(\omega)$ is unitarily equivalent to the Toeplitz $C^{*}$-algebra $C^{*}\left(\left\{T_{Z^{\mu}}: \mu \in \Gamma_{\Omega}\right\}\right)$, so that $C^{*}\left(T_{Z}\right)$ is unitarily equivalent to a natural subalgebra of $\mathscr{W}(\omega)$.

Our next step in trying to describe the structure of $C^{*}\left(T_{Z}\right)$ is to associate to $\omega$ an appropriate groupoid $\mathscr{G}$ so that $\mathscr{W}(\omega)$ becomes a natural subalgebra of $C_{\text {red }}^{*}(\mathscr{G})$ (which, in this case, is isomorphic to $C^{*}(\mathscr{G})$ ). By finding a convenient dissection of $\mathscr{G}$ we will then be able to find a decomposition (groupoid- $C^{*}$-algebra) series for $\mathscr{W}(\omega)$. We illustrate our procedure with an important example.

Remark 2.5. The example of the generalized Hartogs wedge.

(a) The definition. Given $0 \leq \beta<\alpha<\infty$, let

$$
\boldsymbol{\Omega}_{\alpha, \beta}=\left\{(z, w) \in \mathbf{C}^{2}:|z|^{\alpha}<|w|<|z|^{\beta},|w|<1\right\} .
$$

When $\alpha=1, \beta=0$, we obtain the classical Hartogs wedge (or triangle, see [9]). 
(b) Geometrical properties. Observe that $\Omega_{\alpha, \beta}$ is always pseudoconvex. In fact, $\log \left(\Omega_{\alpha, \beta_{+}}\right)$is the wedge in the third quadrant $\mathbf{R}_{-}^{2}$ consisting of the region between the half lines of slopes $\alpha$ and $\beta$, respectively. Notice that $\Omega_{\alpha, \beta}$ is the simplest example of a pseudoconvex Reinhardt domain which is not complete and satisfies condition $(*)$.

(c) Spectral picture. From [19, Theorem 1.3], it follows that

$$
\sigma\left(T_{Z}\right)=\overline{\Omega_{\alpha, \beta}}, \quad \sigma_{e}\left(T_{Z}\right)=\partial \Omega_{\alpha, \beta},
$$

where $\sigma\left(T_{Z}\right)$ and $\sigma_{e}\left(T_{Z}\right)$ denote the joint (Taylor) spectrum and the joint (Taylor) essential spectrum of $T_{Z}$, respectively. One can also check that the polynomial convex hull (and even the Stein-hull [15]) of $\overline{\Omega_{\alpha, \beta}}$ coincides with the closure of the (open) unit polydisk $\mathbf{D}^{2}$. In particular, this implies that $T_{Z}$ is not analytically cyclic on $B^{2}\left(\Omega_{\alpha, \beta}\right)$ (in the sense of [5]).

(d) The weight sequence. One can readily compute the following values for the weight sequence $\left\{\omega(\nu): \nu=\left(\nu_{1}, \nu_{2}\right) \in B_{\Omega_{\alpha, \beta}}\right\}$ :

$$
\begin{aligned}
\omega^{2}(\nu) & =\left\|z^{\nu}\right\|^{2}=(2 \pi)^{2} \int_{0}^{1} r_{1}^{2 \nu_{1}} r_{1} d r_{1} \int_{r_{1}^{\alpha}}^{r_{1}^{\beta}} r_{2}^{2 \nu_{2}} r_{2} d r_{2} \\
& =\frac{\pi^{2}(\alpha-\beta)}{\left[1+\nu_{1}+\alpha\left(1+\nu_{2}\right)\right]\left[1+\nu_{1}+\beta\left(1+\nu_{2}\right)\right]}, \\
1+\nu_{1}+\alpha\left(1+\nu_{2}\right)>0, & 1+\nu_{1}+\beta\left(1+\nu_{2}\right)>0 .
\end{aligned}
$$

Thus, $B_{\Omega_{\alpha, \beta}}^{\prime}=\left\{\rho \in \mathbf{R}^{2}: 1+\rho_{1}+\alpha\left(1+\rho_{2}\right)>0,1+\rho_{1}+\beta\left(1+\rho_{2}\right)>0\right\}$ and $\Gamma_{\Omega_{\alpha, \beta}}^{\prime}=\left\{\sigma \in \mathbf{R}^{2}: \sigma_{1}+\alpha \sigma_{2}>0, \sigma_{1}+\beta \sigma_{2}>0\right\}$, and hence $\Gamma_{\Omega_{\alpha, \beta}}^{\prime}=1+B_{\Omega_{\alpha, \beta}}^{\prime}$.

Definition 2.6. Let now $\Omega$ be an arbitrary pseudoconvex (bounded) Reinhardt domain in $\mathbf{C}^{2}$. We continue using the notation introduced previously. Following the pattern of $[4,19]$, let $\mathscr{A}$ be the $C^{*}$-subalgebra of $l^{\infty}\left(\mathbf{Z}^{2}\right)$ generated by right $\mathbf{Z}^{2}$-translates $\operatorname{Tr}$ of the functions

$$
w_{\mu}(\nu)=\frac{\omega(\mu+\nu) \chi_{B_{\Omega}}(\nu)}{\omega(\nu)}, \quad \mu \in \Gamma_{\Omega}, \nu \in \mathbf{Z}^{2} .
$$

These functions are well defined because

$$
\operatorname{supp}(\omega(\mu+\cdot)) \subseteq \operatorname{supp}(\omega(\cdot)), \quad \forall \mu \in \Gamma_{\Omega} .
$$

(Notice that (13) extends to $\mathbf{Z}^{2}$ the weight sequences defined in (4).) Let $X$ be the maximal ideal space of $\mathscr{A}$. Also, let $\tau: \mathbf{Z}^{2} \mapsto X$ be the map induced by evaluation, i.e.,

$$
\begin{gathered}
\tau(\lambda)\left(\operatorname{Tr}_{\mu} w_{\nu}\right)=w_{\nu}(\mu+\lambda), \\
\lambda, \mu \in \mathbf{Z}^{2}, \quad \nu \in \Gamma_{\Omega} .
\end{gathered}
$$

(Then $\operatorname{Ran}(\tau)$ is dense in $X$, and there is a natural action of $\mathbf{Z}^{2}$ on $X$.) We let $X_{+}$be the closure of $\tau\left(\Gamma_{\Omega}\right)$, and we let $\mathscr{G}$ be the groupoid obtained by reducing the transformation group $X \times \mathbf{Z}^{2}$ (with the canonical left Haar-systems) to $X_{+}$, i.e.

$$
\mathscr{G}=\left.\left(X \times \mathbf{Z}^{2}\right)\right|_{X_{+}}
$$


Remark 2.7. It is easy to see that $\mathscr{K} \subseteq C^{*}(\mathscr{G})$ (see Remark 2.10 below). In fact, $\mathscr{K}$ is $*$-isomorphic to the $C^{*}$-algebra of the reduction groupoid $\mathscr{G}_{\mid \tau\left(\Gamma_{\Omega}\right)}$. Thus, to study $C^{*}(\mathscr{G})$, we need only consider $C^{*}\left[\left(X \times \mathbf{Z}^{2}\right)_{\mid \partial X_{+}}\right]$, where $\partial X_{+}=$ $X_{+} \backslash \tau\left(\Gamma_{\Omega}\right)$. Before we proceed now, we need to introduce a compactification of $\mathbf{R}^{2}$ implicitly used in [19]. This is a regular compactification of $\mathbf{R}^{2}$ in the sense of [11].

Definition 2.8. Let $Y$ be the set $Y=\mathbf{R}^{2} \cup \partial \mathbf{R}^{2}$, where

$$
\partial \mathbf{R}^{2}=\left\{(u, y): u=\left(u_{1}, u_{2}\right) \in \mathbf{T}, y \in \overline{\mathbf{R}} \cdot u^{\perp}\right\},
$$

where $u^{\perp}=\left(-u_{2}, u_{1}\right), \mathbf{T}=\left\{x \in \mathbf{R}^{2}:\|x\|=1\right\}$, and $\overline{\mathbf{R}}=\{-\infty\} \cup \mathbf{R} \cup\{\infty\}$. We need only specify the topology of $Y$ on the (boundary) set $\partial \mathbf{R}^{2}$. Given a pair $(u, y) \in \partial \mathbf{R}^{2}, y \in \mathbf{R} . u^{\perp}$, a neighborhood of $(u, y)$ is the union of two sets of the form

$$
\begin{aligned}
& \mathscr{U}_{\varepsilon}=\left\{z \in \mathbf{R}^{2}:\|z\|>\frac{1}{\varepsilon},\left\|u-\frac{z}{\|z\|}\right\|<\varepsilon,\left\|y-\left\langle z, u^{\perp}\right\rangle u^{\perp}\right\|<\varepsilon\right\}, \\
& \mathscr{V}_{\varepsilon}=\left\{(v, x) \in \partial \mathbf{R}^{2}:\|u-v\|<\varepsilon,\|x-y\|<\varepsilon\right\} .
\end{aligned}
$$

If $y= \pm \infty u^{\perp}$, then a neighborhood of $(u, y)$ is the union of two sets of the form

$$
\begin{array}{r}
\mathscr{W}_{\varepsilon}=\left\{z \in \mathbf{R}^{2}:\|z\|>\frac{1}{\varepsilon},\left\|u-\frac{z}{\|z\|}\right\|<\varepsilon, \quad\left|\left\langle z, u^{\perp}\right\rangle\right|>\frac{1}{\varepsilon},\right. \\
\left.\left\langle z, u^{\perp}\right\rangle= \pm\left|\left\langle z, u^{\perp}\right\rangle\right|\right\}, \\
\mathscr{Z}_{\varepsilon}=\left\{(v, x) \in \partial \mathbf{R}^{2}:\|u-v\|<\varepsilon,\|x\|>\frac{1}{\varepsilon},\left\| \pm u^{\perp}-\frac{x}{\|x\|}\right\|<\varepsilon\right\} .
\end{array}
$$

(Notice that $\mathbf{R}^{2}$ is dense in $Y$.) Let $Y_{+}$be the closure of $\Gamma_{\Omega}$ in $Y$ (of course, $Y_{+}$is then compact and Hausdorff).

As we shall see in the next lemma, $\tau$ is injective on $\Gamma_{\Omega}$ so that $X_{+}$is a regular compactification of $\Gamma_{\Omega}$. We shall also see that $C^{*}(\mathscr{G})$ is $*$-isomorphic to the $C^{*}$-algebra generated by the unweighted forward shifts on $l^{2}\left(B_{\Omega}\right)$ and the multiplication operators by $w_{\nu}, \nu \in \Gamma_{\Omega}$ (see Remark 2.10 below). This will not make use of the hypothesis that $T_{Z}$ is jointly bounded below (as was done in [4, Theorem 2.5]).

Lemma 2.9. The map $\left.\tau\right|_{\Gamma_{\Omega}}$ is injective and extends to a continuous surjection $\tilde{\tau}: Y_{+} \mapsto X_{+}$.

Proof. We recall that the square of $w_{\nu}(\mu): \nu \in \Gamma_{\Omega}, \mu \in B_{\Omega}$ is the $2 \nu$ th momentum of the regular Borel probability measure on $\Omega_{+}$given by

$$
d m(\mu)(r)=\frac{r^{2(\mu+1)} d(\log (r))}{\int_{\Omega_{+}} r^{2(\mu+1)} d(\log (r))} .
$$

On the logarithmic domain, the above measures have the following expression

$$
\log (d m(\mu))(x)=\frac{e^{2\langle\mu+1, x\rangle} d x}{\int_{\log \left(\Omega_{+}\right)} e^{2\langle\mu+1, x\rangle} d x} .
$$


From (15) and (17), it is clear that $\tau$ is injective on $\Gamma_{\Omega}$, and the first assertion follows. We next define the extension $\tilde{\tau}$. Observe that

$$
\partial Y_{+}=Y_{+} \backslash \Gamma_{\Omega}=\left\{(u, y) \in \partial \mathbf{R}^{2}: u \in \mathbf{T} \cap \overline{\Gamma_{\Omega}^{\prime}},\left\langle y, u^{\perp}\right\rangle \in \overline{\left\langle\left\langle\Gamma_{\Omega}, u^{\perp}\right\rangle\right.}\right\} .
$$

Let $(u, y) \in \partial Y_{+}$. Then there exists $\left\{\lambda_{k}\right\} \subseteq \Gamma_{\Omega}$, such that $(u, y)=\lim \lambda_{k}$ in $Y_{+}$. This means that $\lim \lambda_{k} /\left\|\lambda_{k}\right\|=u$ and $\lim \left\langle\lambda_{k}, u^{\perp}\right\rangle u^{\perp}=y$. A very similar argument to the one given in [19, Proposition 3.2], proves that the sequence of measures $\log \left(d m\left(\lambda_{k}\right)\right)$ converges *-weakly to a $\operatorname{limit} \log (d m(u, y))$ that depends only on $(u, y)$. Indeed, let $F_{u}$ be the face of the convex set $C=$ $\log \left(\Omega_{+}\right)$determined by $u$ as the outer normal of $C$ at points of $F_{u}$. If $F_{u}$ is reduced to a point $P \in \partial C$ (i.e., $P$ is an extreme point of $C$ ), then $\log (d m(u, y))$ is the Dirac mass at $P$, and hence it does not depend on $y$. Of course, in this case, there may be more than one $u$ that corresponds to the same limit (for example, if $P$ is a sharp vertex of $C$ ). If $F_{u}$ is one-dimensional but $y= \pm \infty u^{\perp}$, then again $\log (d m(u, y))$ is the Dirac mass at $P$, where $P$ is one of the extreme points of $F_{u}$. If we orient $\partial C$ positively, then, as we travel throughout $\partial C$ in the positive direction, $P$ is the first extreme point $P_{-}$ of $F_{u}$ if $y=-\infty u^{\perp}$, and it is the second extreme point $P_{+}$if $y=\infty u^{\perp}$. If $|y|<\infty$, then

$$
\log (d m(u, y))=\frac{e^{2\left\langle y+1, u^{\perp}\right\rangle x} d m_{F}(x)}{\int_{F} e^{2\left\langle y+1, u^{\perp}\right\rangle x} d m_{F}(x)},
$$

where $d m_{F}$ is the linear Lebesgue measure on $F=F_{u}$. It follows now easily (just as in [19, Proposition 3.2]) from (15) that, if either $F_{u}$ is an extreme point $P$ of $C=\log \left(\Omega_{+}\right)$, or if $F_{u}$ is one-dimensional but $|y|=\infty$ (so that $(u, y)$ also corresponds to an extreme point $P$ of $C)$, then $(\tilde{\tau}(u, y))\left(\operatorname{Tr}_{\mu} w_{\nu}\right)=$ $\operatorname{Exp}(\langle\nu, P\rangle)$ for every $y \in \mathbf{R} u^{\perp}$ and every $\mu \in \mathbf{Z}^{2}$. On the other hand, if $F_{u}$ is one-dimensional and $|y|<\infty$, then for $\nu \in \Gamma_{\Omega}, \mu \in \mathbf{Z}^{2},\left\langle y+\mu+\nu+1, u^{\perp}\right\rangle \neq$ 0 , and $\left\langle y+\mu, u^{\perp}\right\rangle$ is the closure of the projection of $\Gamma_{\Omega}$ onto $\overline{\mathbf{R}} u^{\perp}$, we have (18)

$$
\begin{aligned}
& {\left[(\tilde{\tau}(u, y))\left(\operatorname{Tr}_{\mu} w_{\nu}\right)\right]^{2}=\frac{\int_{F} e^{2\left\langle y+1+\mu+\nu, u^{\perp}\right\rangle x} d m_{F}(x)}{\int_{F} e^{2\left\langle\mu+y+1, u^{\perp}\right\rangle x} d m_{F}(x)}} \\
& \quad=\frac{\left\langle y+\mathbf{1}+\mu, u^{\perp}\right\rangle}{\left\langle y+\mathbf{1}+\mu+\nu, u^{\perp}\right\rangle} \frac{e^{2\left\langle y+1+\mu+\nu, u^{\perp}\right\rangle\left\langle u^{\perp}, P_{+}\right\rangle}-e^{2\left\langle y+1+\mu+\nu, u^{\perp}\right\rangle\left\langle u^{\perp}, P_{-}\right\rangle}}{e^{2\left\langle y+1+\mu, u^{\perp}\right\rangle\left\langle u^{\perp}, P_{+}\right\rangle}-e^{2\left\langle y+1+\mu, u^{\perp}\right\rangle\left\langle u^{\perp}, P_{-}\right\rangle}},
\end{aligned}
$$

where $P_{+}$and $P_{-}$are the extreme points of $F_{u}$ as above. From (18) we see that $\tilde{\tau}$ is continuous (see the proof of [19, Lemma 3.3]). In order to prove surjectivity, let $x \in \partial X_{+}=X_{+} \backslash \tau\left(\Gamma_{\Omega}\right)$. Then there exists $\left\{\lambda_{k}\right\} \subseteq \Gamma_{\Omega}$ such that $\lim \tau\left(\lambda_{k}\right)=x$. By dropping to a subsequence, if necessary, we can assume that

$$
\lim \left\|\lambda_{k}\right\|=\infty, \quad \lim \frac{\lambda_{k}}{\left\|\lambda_{k}\right\|}=u, \quad \lim \left\langle\lambda_{k}, u^{\perp}\right\rangle u^{\perp}=y,
$$

for some $u \in \mathbf{T}, y \in \overline{\mathbf{R}} u^{\perp}$. But, then $\tilde{\tau}(u, y)=x$, as desired.

Remark 2.10. (a) Now we turn our attention to groupoid $C^{*}$-algebras. It follows readily that the transformation group $C^{*}$-algebra $C^{*}\left(X \times \mathbf{Z}^{2}\right)$ is isomorphic to the $C^{*}$-algebra generated by the translation (bilateral shift) operators $\left\{V_{\mu}: \mu \in\right.$ $\left.\mathbf{Z}^{2}\right\}$, and the diagonal operators of weight sequence $\left\{w_{\nu}: \nu \in \Gamma_{\Omega}\right\}$ (of (13), all acting on $\mathscr{L}\left(l^{2}\left(\mathbf{Z}^{2}\right)\right)$. 
(b) Let $U_{\nu}$ be the unilateral shift consisting of the restriction to $\mathscr{L}\left(l^{2}\left(B_{\Omega}\right)\right)$ of $V_{\nu, \nu \in \Gamma_{\Omega}}$. Also, let $D_{\nu}$ be the diagonal operator on $\mathscr{L}\left(l^{2}\left(B_{\Omega}\right)\right)$ with weight sequence given by (4), $\nu \in \Gamma_{\Omega}$. It follows easily that $C_{\text {red }}^{*}(\mathscr{G})$ is isomorphic to the $C^{*}$-algebra generated by $\left\{\left(U_{\nu}, D_{\nu}\right): \nu \in \Gamma_{\Omega}\right\}$. Notice that $C_{\text {red }}^{*}(\mathscr{G}) \simeq$ $C^{*}(\mathscr{G})$ in our case, because $\mathscr{G}$ is amenable [11, §2]. Therefore, since

$$
W_{\phi \nu}=U_{\nu} D_{\nu} \simeq S_{\nu} \simeq T_{Z^{\nu}}, \quad \nu \in \Gamma_{\Omega},
$$

we conclude that $\mathscr{W}(\omega)$ is isomorphic to a natural subalgebra of $C^{*}(\mathscr{G})$, namely $C^{*}\left(\left\{U_{\nu} D_{\nu}: \nu \in \Gamma_{\Omega}\right\}\right)$.

(c) Assume now that $\Omega \subseteq \mathbf{C}^{n}$, and let $U_{+}$be the $n$-tuple of shifts $U_{\nu}$ where $\nu$ runs through the $n$ multi-indices in $\mathrm{Z}_{+}^{n}$ of modulus 1 (i.e., corresponding to shifting in each coordinate). We claim that $C^{*}\left(U_{+}\right)=C^{*}\left(\left\{U_{\nu}: \nu \in \Gamma_{\Omega}\right\}\right)$. Since $\mathbf{Z}_{+}^{n} \subseteq \Gamma_{\Omega}$, in order to prove this claim it is enough to show that $U_{\nu} \in C^{*}\left(U_{+}\right)$ for a given $\nu \in \Gamma_{\Omega}$. Let $\kappa, \lambda \in \mathbf{Z}_{+}^{n}$, such that $\nu=\kappa-\lambda$. Then, we have $U_{\lambda} U_{\nu}=U_{\lambda+\nu}=U_{\kappa}$. But, $U_{\lambda}$ is an isometry (because $B_{\Omega}$ is invariant under translations of elements in $\Gamma_{\Omega}$ ), so

$$
U_{\nu}=U_{\lambda}^{*} U_{\kappa}=\left(U_{+}^{*}\right)^{\lambda} U_{+}^{\kappa},
$$

as desired. An immediate consequence of the above claim is that

$$
C^{*}\left(\left\{\left(U_{\nu}, D_{\nu}\right): \nu \in \Gamma_{\Omega}\right\}\right)=C^{*}\left(U_{+},\left\{D_{\nu}: \nu \in \Gamma_{\Omega}\right\}\right) \text {. }
$$

Let $D_{+}$be the $n$-tuple of diagonal operators $D_{\nu}$, where $\nu$ runs over the multiindices of modulus 1 in $\mathbf{Z}_{+}^{n}$. By the canonical representation of

$$
C^{*}\left(\left\{T_{Z}^{\nu}: \nu \in \Gamma_{\Omega}\right\}\right) \simeq \mathscr{W}(\omega),
$$

we deduce that $T_{Z}=U_{+} D_{+}$(the product here is componentwise) is the polar decomposition of $T_{Z}$. Therefore, $C^{*}\left(T_{Z}\right)$ is also isomorphic to a natural subalgebra of $C^{*}(\mathscr{G})$.

In order to describe a good parameterization of the groupoid $\mathscr{G}$ that allows us to perform surgery on $X_{+}$, we shall define a compact Hausdorff space $Z_{+}$ (which is naturally related to the geometry of $\partial \log \left(\Omega_{+}\right)$) and a map $q: \partial Y_{+}=$ $Y_{+} \backslash \Gamma_{\Omega} \rightarrow Z_{+}$such that $\tilde{\tau}$ is constant exactly where $q$ is constant, so that the map $\hat{\tau}: Z_{+} \rightarrow \partial X_{+}$induced by $\tilde{\tau}$ is covariant and injective, i.e., a covariant homeomorphism.

Remark 2.11. We consider again the example of the Hartogs wedge, to illustrate our methods. If $\Omega=\Omega_{\alpha, \beta}$, then 18 reduces to the following. Let

$$
\mu \in \mathbf{Z}^{2}, \quad \nu \in \Gamma_{\Omega}, \quad(u, y) \in Y_{+} .
$$

If $u \in \operatorname{int}\left(\Gamma_{\Omega_{\alpha, \beta}}^{\prime}\right)$, then

$$
\left[(\tilde{\tau}(u, y))\left(\operatorname{Tr}_{\mu} w_{\nu}\right)\right]^{2}=1, \quad y \in \overline{\mathbf{R}} u^{\perp} .
$$

(This is because $\log (d m(u, y))$ is the Dirac mass at the vertex $(0,0)$ of the cone $\log \left(\Omega_{\alpha, \beta_{+}}\right)$.) If

$$
u=u_{\alpha}=\frac{(\alpha,-1)}{\left(1+\alpha^{2}\right)^{1 / 2}},
$$

then we have

$$
\begin{aligned}
{\left[(\tilde{\tau}(u, y))\left(\operatorname{Tr}_{\mu} w_{\nu}\right)\right]^{2} } & =\frac{\left\langle y+\mathbf{1}+\mu, u^{\perp}\right\rangle}{\left\langle y+1+\mu+\nu, u^{\perp}\right\rangle}, \quad\left\langle y+\mathbf{1}+\mu, u^{\perp}\right\rangle>0, \\
(\tilde{\tau}(u, y))\left(\operatorname{Tr}_{\mu} w_{\nu}\right) & =0, \quad\left\langle y+\mathbf{1}+\mu, u^{\perp}\right\rangle \leq 0 .
\end{aligned}
$$


On the other hand, if

$$
u=u_{\beta}=\frac{(-\beta, 1)}{\left(1+\beta^{2}\right)^{1 / 2}}
$$

then we have

$$
\begin{aligned}
{\left[(\tilde{\tau}(u, y))\left(\operatorname{Tr}_{\mu} w_{\nu}\right)\right]^{2} } & =\frac{\left\langle y+\mathbf{1}+\mu, u^{\perp}\right\rangle}{\left\langle y+\mathbf{1}+\mu+\nu, u^{\perp}\right\rangle}, \quad\left\langle y+\mathbf{1}+\mu, u^{\perp}\right\rangle<0, \\
(\tilde{\tau}(u, y))\left(\operatorname{Tr}_{\mu} w_{\nu}\right) & =0, \quad\left\langle y+\mathbf{1}+\mu, u^{\perp}\right\rangle \geq 0 .
\end{aligned}
$$

Thus, our candidate for the set $\mathbf{Z}_{+}$is the disjoint union of the vertex $V$ of the cone $\log \left(\Omega_{+}\right)$, and the closure of the projections of $\Gamma_{\Omega}$ onto $\mathbf{R} u_{\alpha}^{\perp}$ (i.e., $[0, \infty) u_{\alpha}^{\perp}$ if $\alpha$ is irrational), and onto $\mathbf{R} u_{\beta}^{\perp} \mathbf{R}$ (i.e., $(-\infty, 0] u_{\beta}^{\perp}$ if $\beta$ is irrational). Let $\mathscr{I}$ be the commutator ideal of $C^{*}(\mathscr{G})$. Then we have

$$
\begin{gathered}
C^{*}\left(\left.\mathscr{G}\right|_{\{V\}}\right) \simeq C^{*}(\mathscr{G}) / \mathscr{I} \simeq C\left(\mathbf{T}^{2}\right), \\
C^{*}\left(\left.\mathscr{G}\right|_{Z_{+} \backslash\{V\}}\right) \simeq \mathscr{I} / \mathscr{K} \simeq \mathscr{J}_{\alpha} \oplus \mathscr{I}^{\beta},
\end{gathered}
$$

where $\mathscr{J}_{\alpha}$ and $\mathscr{I}^{\beta} \simeq \mathscr{I}_{\beta}$ are groupoid $C^{*}$-algebras described as follows. Given a real number $\gamma$, let $\mathscr{X}$ be the closure in $\mathbf{R}$ of the subgroup $\{m+\gamma n:(m, n) \in$ $\left.\mathbf{Z}^{2}\right\}$ (so that if $\gamma$ is irrational, then $\mathscr{X}=\mathbf{R}$ ). Also, let $\mathscr{X} \otimes^{\gamma} \mathbf{Z}^{2}$ and $\mathscr{X} \otimes_{\gamma} \mathbf{Z}^{2}$ be the transformation group spaces obtained by the actions of $\mathbf{Z}^{2}$ on $\mathscr{X}$ defined by $(x, m, n) \rightarrow x-m-\gamma n$ and $(x, m, n) \rightarrow x+m+\gamma n$, respectively. Let

$$
\mathscr{G}_{\gamma}=\left(\mathscr{X} \otimes_{\gamma} \mathbf{Z}^{2}\right)_{\mid \mathscr{X} \cap[0, \infty)}, \quad \mathscr{G}^{\gamma}=\left(\mathscr{X} \otimes^{\gamma} \mathbf{Z}^{2}\right)_{\mid \mathscr{X} \cap(-\infty, 0]},
$$

then $\mathscr{I}_{\alpha}$ and $\mathscr{I}^{\beta}$ are the $C^{*}$-algebras associated with the groupoids $\mathscr{G}_{\alpha}$ and $\mathscr{G}^{\beta}$, respectively. If either $\alpha$ or $\beta$ is irrational, then $C^{*}(\mathscr{G})$ is not of type $\mathrm{I}$. We will see in just a moment that the same conclusion holds for the $C^{*}$-subalgebras $\mathscr{W}(\omega)$ and $C^{*}\left(T_{Z}\right)$. First observe that an easy manipulation with weights shows that the diagonal positive operators $D_{\nu}$ and $D_{+, j}$ (i.e., the absolute values of the operators $T_{Z^{\nu}}$ and $T_{Z_{j}}$ ) are bounded below, $\nu \in \Gamma_{\Omega_{\alpha, \beta}}, 1 \leq j \leq$ $n$. This readily implies that $\mathscr{W}(\omega)=C^{*}(\mathscr{G}) \simeq C^{*}\left(T_{Z}\right)$.

Definition 2.12. Given $\Omega \subseteq \mathbf{C}^{2}$, let $C$ be the (open) convex set $C=\log \left(\Omega_{+}\right)$, and let $\mathbf{F}(C)$ be the set of all open faces in $\partial C$. Here, we include in $\mathbf{F}(C)$ a possible $F$ that is a virtual face of $C$, i.e., $F$ is tangent to $\partial C$ at $\infty$ (see $[19, \S 3])$. We let $Z_{+}$be the set of pairs $(F, z)$ where $F \in \mathbf{F}(C)$ and $z$ is an element of the affine space $[F]$ generated by $F$, which is an appropriate translate of the projection of $\overline{\Gamma_{\Omega}}$ onto $\overline{\mathbf{R}} u^{\perp}$ if $F=F_{u}$ is one-dimensional, and $z=0$. If $F$ is zero-dimensional (i.e., an extreme point), let $q: \partial Y_{+} \rightarrow Z_{+}$be the surjective map defined by $q(u, y)=\left(F_{u}, c u+\left\langle y, u^{\perp}\right\rangle u^{\perp}\right)$, where $F_{u}$ is the face in $\partial C$ determined by $u$ (as an exterior normal), and $c=\sup _{x \in C}\langle u, x\rangle$.

Proposition 2.13. The maps $q$ and $\tilde{\tau}$ define the same level sets on $\partial Y_{+}$. Let $\hat{\tau}$ be the map induced by $\tilde{\tau}$ on $Z_{+}$. Then the space $Z_{+}$(with the quotient topology induced from $\left.\partial Y_{+}\right)$is compact and Hausdorff, and the map $\tilde{\tau}: Z_{+} \rightarrow \partial X_{+}$is a covariant homeomorphism. Furthermore, if either $F=F_{u}$ is an extreme point $P$ of $C=\log \left(\Omega_{+}\right)$(so that $z=0$ ), or if $F_{U}$ is one-dimensional but $|z|=\infty$ (so that $(F, z)$ also corresponds to an extreme point $P$ of $C$ ), then

$$
(\hat{\tau}(F, z))\left(\operatorname{Tr}_{\mu} w_{\nu}\right)=\operatorname{Exp}(\langle\nu, P\rangle)
$$


for every $\mu \in \mathbf{Z}^{2}$. On the other hand, if $F_{u}$ is one-dimensional and $|z|<\infty$, then for $\nu \in \Gamma_{\Omega}, \mu \in \mathbf{Z}^{2},\left\langle z+\mathbf{1}+\mu+\nu, u^{\perp}\right\rangle \neq 0$, and $\left\langle z+\mu, u^{\perp}\right\rangle$ in the closure of the projection of $\Gamma_{\Omega}$ onto $\overline{\mathbf{R}} u^{\perp}, \hat{\tau}$ is given by

$$
\begin{aligned}
& {\left[(\hat{\tau}(F, z))\left(\operatorname{Tr}_{\mu} w_{\nu}\right)\right]^{2}=\frac{\int_{F} e^{2\left\langle z+\mathbf{1}+\mu+\nu, u^{\perp}\right\rangle x} d m_{F}(x)}{\int_{F} e^{2\left\langle z+1+\mu, u^{\perp}\right\rangle x} d m_{F}(x)}} \\
& \quad=\frac{\left\langle z+\mathbf{1}+\mu, u^{\perp}\right\rangle}{\left\langle z+\mathbf{1}+\mu+\nu, u^{\perp}\right\rangle} \frac{e^{2\left\langle z+1+\mu+\nu, u^{\perp}\right\rangle\left\langle u^{\perp}, P_{+}\right\rangle}-e^{2\left\langle z+1+\mu+\nu, u^{\perp}\right\rangle\left\langle u^{\perp}, P_{-}\right\rangle}}{e^{2\left\langle z+1+\mu, u^{\perp}\right\rangle\left\langle u^{\perp}, P_{+}\right\rangle}-e^{2\left\langle z+1+\mu, u^{\perp}\right\rangle\left\langle u^{\perp}, P_{-}\right\rangle}},
\end{aligned}
$$

where $d m_{F}$ is the (linear) Lebesgue measure on $F=F_{u}$, and $P_{+}$and $P_{-}$are the extremes points of $F_{u}$.

Proof. The fact that $\tilde{\tau}$ is constant on the level sets of $q$ follows immediately from the definition of $Z_{+}$and formula (18) of the proof of Lemma 2.9. Since $\tilde{\tau}=\hat{\tau} \circ q$, the continuity of $\hat{\tau}$ is a consequence of the continuity of $q$ and $\tilde{\tau}$. Thus, in order to complete the proof, it clearly suffices to show that $\hat{\tau}$ is bijective and covariant. But, the fact that $\hat{\tau}$ is injective follows from a periodicity argument very similar to the one given in the proof of [19, Lemma 3.6]. Since $\tilde{\tau}$ is surjective, by Lemma $2.9, \hat{\tau}$ is also surjective. Finally, since $\tilde{\tau}$ and $q$ are obviously covariant, it follows that $\hat{\tau}$ is covariant.

Theorem 2.14. Let $\mathscr{G}$ be the groupoid constructed from the reduction of $X \times \mathbf{Z}^{2}$ to $X_{+}$, as above. Further, let $\mathscr{I}$ be the commutator ideal in $C^{*}(\mathscr{G})$. Then $\mathscr{K} \subseteq \mathscr{I}$,

$$
\frac{C^{*}(\mathscr{G})}{\mathscr{K}} \simeq C^{*}\left(\mathscr{G}_{\mid \partial X_{+}}\right), \quad \frac{C^{*}(\mathscr{G})}{\mathscr{I}} \simeq C(\operatorname{Extreme}(\Omega)), \quad \frac{\mathscr{I}}{\mathscr{K}} \simeq \bigoplus_{F} C^{*}(F),
$$

where the above direct sum runs over all the one-dimensional faces $F \in$ $\mathscr{F}\left(\overline{\left.\log \left(\Omega_{+}\right)\right)}\right.$and $\operatorname{Extreme}(\Omega)$ is the closure of $\bar{\Omega}$ of the subset of $\Omega$ whose positive part is the image under the exponential map of the set of extreme points of $\log \left(\Omega_{+}\right)$, and $C^{*}(F)$ is the groupoid $C^{*}$-algebra defined as follows. Let $u=\left(u_{1}, u_{2}\right) \in \mathbf{T}$ such that $F=F_{u}$, and let $u^{\perp}=\left(-u_{2}, u_{1}\right)$. Also, let $\mathscr{Y}$ be the closure in $\mathbf{R}$ of

$$
\left\langle\mathbf{Z}^{2}, u^{\perp}\right\rangle=\left\{-\nu_{1} u_{2}+\nu_{2} u_{1}: \nu=\left(\nu_{1}, \nu_{2}\right) \in \mathbf{Z}^{2}\right\},
$$

and let $\mathscr{Y} \times \mathbf{Z}^{2}$ be the transformation group induced by the action $(y, m, n) \rightarrow$ $y+n u_{1}-m u_{2}$. Then $C^{*}(F)$ is the $C^{*}$-algebra of the groupoid obtained by reducing $\mathscr{Y} \times \mathbf{Z}^{2}$ to the closure of the projection of $\Gamma_{\Omega}$ onto $\mathscr{Y}$ i.e., to $\overline{\left\langle\Gamma_{\Omega}, u^{\perp}\right\rangle}$. (Notice that this projection coincides with $\mathscr{Y}$ unless that $F$ is a virtual face.)

Proof. The proof uses Lemma 2.9, Proposition 2.13, and the same argument as [19, Theorem 4.3].

The following theorem can be proved using the same techniques.

Theorem 2.15. Let $\Omega$ be a (bounded) pseudoconvex Reinhardt domain in $\mathbf{C}^{n}$, and assume that $\partial \Omega$ contains no analytic disk, i.e., $\partial \Omega=\operatorname{Extreme}(\Omega)$. Then $\mathscr{K}$ coincides with the commutator ideal $\mathscr{J}$ of $C^{*}\left(1, T_{Z}\right)$, and $C^{*}\left(1, T_{Z}\right) / \mathscr{J} \simeq$ $C(\partial \Omega)$.

Remark 2.16. (a) Throughout the rest of this section, $\Omega$ will be a (bounded) pseudoconvex Reinhardt domain in $\mathbf{C}^{n}$ (where $n$ is arbitrary), and we let $\mu$ be 
a Reinhardt regular Borel positive finite measure on $\Omega$, i.e., $\mu=\lambda \times \theta$, where $\lambda$ is a regular Borel positive finite measure whose support is contained in $\overline{\Omega_{+}}$, and $\theta$ is the standard normalized Haar measure on $\mathbf{T}^{N}$. Also, let $B^{2}(\Omega, \mu)$ and $H^{2}(\Omega, \mu)$ be the subspace of holomorphic functions on $\Omega$ which are in $L^{2}(\mu)$ and the closure in $L^{2}(\mu)$ of $H^{\infty}(\Omega)$, respectively. When $\mu$ is the volume Lebesgue measure on $\Omega$, there is little difference between the spectral and $C^{*}$ algebraic properties of $T_{Z}$ acting on either $B^{2}(\Omega, \mu)$ or $H^{2}(\Omega, \mu)$. As in the case of the Lebesgue measure, a standard basis for $H^{2}(\Omega, \mu)$ on which $\left\{T_{Z}^{\nu}: \nu \in \Gamma_{\Omega}\right\}$ can be expressed as a weighted shift is given by

$$
e_{\nu}(z)=z^{\nu} /\left\|z^{\nu}\right\|, \quad \nu \in \Gamma_{\Omega}
$$

If $\Omega$ satisfies condition $(*)$ (see Lemma 2.2 ), then the $C^{*}$-algebras generated by the canonical $n$-tuples $T_{Z}$ acting on both spaces are $*$-isomorphic. However, when $\mu$ is allowed to have holomorphic functions in $B^{2}(\Omega, \mu)$ which have arbitrary growth near some points of $\partial \Omega$, then the corresponding weighted Wiener-Hopf $C^{*}$-algebras might be quite different. For example, let $\mu$ be the measure on the Hartogs wedge $\Omega=\Omega_{\alpha, \beta}, 0<\alpha<\beta$, given by $\mu=\delta_{(1,1)} \times \theta$, where $\delta_{(1,1)}$ is the Dirac measure at $(1,1)$. Since $Z^{\nu}$ is holomorphic on $\Omega$ and belongs to $L^{2}(\mu)$, for every $\nu \in \mathbf{Z}^{2}$, it follows that $B^{2}(\Omega, \mu)=L^{2}(\mu)=$ $L^{2}\left(\mathbf{T}^{2}\right)$, and the corresponding Wiener-Hopf $C^{*}$-algebra is unitarily equivalent to $C\left(\mathbf{T}^{2}\right)$. On the other hand, $H^{2}(\Omega, \mu)$ is the space of holomorphic functions with Fourier coefficients supported on $\Gamma_{\Omega_{\alpha, \beta}}$, and the corresponding Wiener-Hopf $C^{*}$-algebra is the standard (unweighted) Wiener-Hopf $C^{*}$ algebra on $\Gamma_{\Omega_{\alpha, \beta}}$ (see for example [13]). Thus, in considering the general case of Reinhardt measures $\mu$ on $\Omega$, in order to take into account the geometry of $\partial \Omega$, we can either impose some restrictions on $\mu$ including the condition that $\partial \Omega \subseteq \operatorname{supp}(\mu)$ or we can study $T_{Z}$ only on $H^{2}(\Omega, \mu)$. This latter alternative choice is what we shall make in the sequel.

(b) As in the case when $\mu$ is the Lebesgue measure on $\Omega$, it follows that the Toeplitz $C^{*}$-algebra $C^{*}\left(\left\{T_{z}^{\nu}: \nu \in \Gamma_{\Omega}\right\}\right)$, acting on $H^{2}(\Omega, \mu)$, is *-isomorphic to the Wiener-Hopf $C^{*}$-algebra $\mathscr{W}(\Gamma, \omega)$ acting on $l^{2}(\Gamma, \omega)$, where $\Gamma=\Gamma_{\Omega}$, $\omega(\nu)=\left\|z^{\nu}\right\|_{2, \mu}, \nu \in \Gamma$, and $l^{2}(\Gamma, \omega)$ is the Hilbert space of square-summable sequences with weight function $\omega$ supported on $\Gamma$. Further, under some reasonable hypotheses on $\mu$, there is a natural groupoid $\mathscr{G}(\Omega)$ associated with $(\Gamma, \omega)$ that can be parameterized in terms of $\partial \Omega$, just as we saw previously in the case when $\mu$ is the volume Lebesgue measure and $n=2$. Also, we shall see that $\mathscr{K} \subseteq \mathscr{W}(\Gamma, \omega)$, and $\mathscr{W}(\Gamma, \omega) / \mathscr{K}$ is $*$-isomorphic to a canonical subalgebra of $C_{\text {red }}^{*}(\mathscr{G}(\Omega))=C^{*}(\mathscr{G}(\Omega))$ and a natural decomposition series of this latter algebra can be computed using such a parameterization.

The following theorem gives a more explicit relation between weighted WienerHopf operators in $\mathscr{W}(\Gamma, \omega)$, and Toeplitz operators in $C^{*}\left(\left\{T_{z}^{\nu}: \nu \in \Gamma\right\}\right)$ acting on $H^{2}(\Omega, \mu)$. This result can be considered as a generalization of the classical Berger's Theorem on subnormal weighted shifts. Notice that the forward shifts in the statement of part (a) are not forward shift in the conventional sense, but rather they are forward shift in the direction of the cone $\Gamma$.

Theorem 2.17. Let $\Gamma \subseteq \mathbf{Z}^{n}$ be a pointed positive cone of $\mathbf{Z}^{n}$ containing $\mathbf{Z}_{+}^{n}$. Also, let $\omega: \Gamma \mapsto \mathbf{R}_{+}$be a quasidecreasing strictly positive function, i.e., 


$$
\zeta(\mu)=\sup _{\nu \in \Gamma} \frac{\omega(\mu+\nu)}{\omega(\nu)}<\infty, \quad \forall \mu \in \Gamma .
$$

Then the following statements are equivalent:

(a) There exists a commutative normal semigroup extension of the semigroup $W_{\nu}: \nu \in \Gamma$ of unweighted "forward" shifts, in $\mathscr{L}\left(l^{2}(\Gamma, \omega)\right)$, i.e., this is a subnormal semigroup.

(b) There exists a regular Borel positive finite measure $\lambda$ with compact support in $\mathbf{R}_{+}^{n}$ such that, for every $\nu \in \Gamma$, we have

$$
\omega(\nu)=\left\|R^{\nu}\right\|_{2, \lambda}=\left[\int\left(r^{\nu}\right)^{2} d \lambda(r)\right]^{1 / 2}, \quad \zeta(\nu)=\left\|R^{\nu}\right\|_{\infty, \lambda} .
$$

Proof. In order to prove that (b) implies (a), let $\lambda$ be as in (b), and observe that $R^{\nu}$ is in $L^{\infty}\left(\Omega_{+}\right)$for every $\nu \in \Gamma$, where

$$
\Omega_{+}=\operatorname{Exp}\{\operatorname{conv}[-\widehat{\Gamma} \cup \log (\operatorname{supp}(\lambda))]\} .
$$

Let $\mu$ be the measure induced by $\lambda$ on

$$
\Omega=\left\{\left(t_{1} r_{1}, \ldots, t_{n} r_{n}\right):\left(r_{1}, \ldots, r_{n}\right) \in \Omega_{+},\left|t_{j}\right|=1,1 \leq j \leq n\right\} .
$$

From the condition satisfied by $\lambda$ in (b), we see that the operators $W_{\nu}=$ $T_{Z^{\nu}}: \nu \in \Gamma$, acting on $H^{2}(\Omega, \mu)$, are bounded, and hence $W_{\nu}: \nu \in \Gamma$ is a commutative subnormal semigroup of "forward weighted" shifts, and (a) follows. Conversely, assume that (a) holds, i.e., suppose that $T_{\nu}: \nu \in \Gamma$ is a commuting normal representation on a Hilbert space $\mathscr{H}$ of $\Gamma$ such that $T_{0}=1_{\mathscr{L}}$ and such that $W_{\nu}$ is the restriction of $T_{\nu}$ to $l^{2}(\Gamma, \omega), \forall \nu \in \Gamma$. In particular, the $n$-tuple, denoted by $\mathbf{s}$, whose $j$ th component is $W_{[j]}$ is subnormal with normal extension $\mathbf{t}=\left(T_{[1]}, \ldots, T_{[n]}\right)$ (here, $[j]$ denotes the multi-index with 1 in the $j$ th slot and zero in the other slots). We claim that the commutant $\{\mathbf{s}\}^{\prime}$ of $\mathbf{s}$ is abelian. Indeed, we first determine the commutant $\mathscr{C}$ of $\left\{W_{\nu}: \nu \in \Gamma\right\}$. To this end, let $T \in \mathscr{C}$, and let $T f_{0}=\sum_{\alpha \in \Gamma} a_{\alpha} f_{\alpha}$, where $f_{\alpha}$ is the unit mass at $\alpha \in \Gamma$ and $\left\{a_{\alpha}\right\} \in l^{2}(\Gamma, \omega)$. Then, $\forall \nu \in \Gamma$, we have

$$
\begin{aligned}
T f_{\nu} & =T W_{\nu} f_{0}=W_{\nu} T f_{0}=W_{\nu} \sum_{\alpha \in \Gamma} a_{\alpha} f_{\alpha} \\
& =\sum_{\alpha \in \Gamma} a_{\alpha} f_{\alpha+\nu}=\sum_{\alpha \in \Gamma} a_{\alpha} W_{\alpha} f_{\nu} .
\end{aligned}
$$

We must impose further restrictions to $\left\{a_{\alpha}\right\}$, by asking that for every $x=$ $\sum_{\beta \in \Gamma} b_{\beta} f_{\beta}$ in $l^{2}(\Gamma, \omega)$, the representation

$$
T x=\sum_{\alpha \in \Gamma} a_{\alpha} W_{\alpha} \sum_{\beta \in \Gamma} b_{\beta} f_{\beta}=\sum_{\alpha, \beta \in \Gamma, \alpha+\beta \in \Gamma} a_{\alpha} b_{\beta} f_{\alpha+\beta},
$$

be valid. In any case, since $W_{\nu} \in\{\mathbf{s}\}^{\prime}, \forall \nu \in \Gamma$, it follows that $\{\mathbf{s}\}^{\prime}$ is abelian, establishing our claim. As an immediate consequence we see that the vector $f_{0}$ is cyclic for $\{\mathbf{s}\}^{\prime \prime}\left(=\{\mathbf{s}\}^{\prime}\right)$. We can assume, without loss of generality, that $T_{\nu}: \nu \in \Gamma$ is minimal in the sense that $e_{0}$ is cyclic for the *-algebra generated by $\left\{T_{\nu}: \nu \in \Gamma\right\}$ on $\mathscr{H}$. In view of the above remark, $\left\{T_{\nu}: \nu \in\right.$ 
$\Gamma\} \subseteq\{\mathbf{t}\}^{\prime \prime}$, and hence $e_{0}$ is cyclic for $\{\mathbf{t}\}^{\prime \prime}\left(=\{\mathbf{t}\}^{\prime}\right)$ on $\mathscr{H}$. The positive linear functional on $C(\sigma(\mathbf{t}))$ defined by $g \mapsto\left\langle g(\mathbf{t}) e_{0}, e_{0}\right\rangle$ induces a regular Borel positive finite measure $\mu$ with compact support in $\mathbf{C}^{n}$, namely $\operatorname{supp}(\mu)=\sigma(\mathbf{t})$. The map $g \mapsto g(\mathbf{t})$ extends to a $*$-isomorphism from $L^{\infty}(\mu)$ onto $\{\mathbf{t}\}^{\prime}$. This isomorphism is implemented by the unitary transformation $V: L^{2}(\mu) \mapsto \mathscr{H}$ defined on the dense subset $C(\sigma(\mathbf{t}))$ by $g \mapsto g(\mathbf{t}) f_{0}$. It easily follows that $V\{\mathbf{t}\}^{\prime} V^{*}=L^{\infty}(\mu)$, and that $V \mathbf{t}^{\nu} V^{*}$ is the operator multiplication by $Z^{\nu}$ on $L^{2}(\mu)$. In particular, $Z^{\nu} \in L^{\infty}(\mu), \forall \nu \in \Gamma$. Since $\mathbf{s}$ is unitarily equivalent to $\xi \mathbf{s}=\left(\xi_{1} W_{[1]}, \ldots, \xi_{n} W_{[n]}\right)$ for every $\xi$ in the $n$th torus $\mathbf{T}^{n}$, we deduce that $\operatorname{supp}(\mu)$ is also invariant under the action of $\mathrm{T}^{n}$, i.e., $\mu$ is a Reinhardt measure. Thus, we can write $\mu$ as $\mu=\lambda \times \theta$, where $\lambda$ is a regular Borel positive finite measure with compact support in $\mathbf{R}_{+}^{n}$, and $\theta$ is the normalized Haar measure on $\mathbf{T}^{n}$. Therefore, this forces $R_{\nu} \in L^{\infty}(\lambda)$, for every $\nu \in \Gamma$. We also have

$$
(\omega(\nu))^{2}\left\|W_{\nu} f_{0}\right\|^{2}=\left\|T_{\nu} f_{0}\right\|^{2}=\left\|Z^{\nu}\right\|^{2}=\int\left(r^{\nu}\right)^{2} d \lambda(r) .
$$

Since $\left\|W_{\nu}\right\|=\zeta(\nu)$, and obviously $\zeta(\nu) \leq\left\|R^{\nu}\right\|_{\infty, \lambda}=\left\|T_{Z^{\nu}}\right\|=\left\|W_{\nu}\right\|$, for every $\nu \in \Gamma$, the proof of the theorem is complete.

Remark 2.18. (a) If the weight function $\omega$ is actually decreasing, i.e., $\zeta(\nu)=1$, $\forall \nu \in \Gamma$, then, from the previous proof, we see that $\operatorname{supp}(\lambda) \subseteq-\widehat{\Gamma}$. In any case, the operator $W_{\nu}$ is unitarily equivalent to the Toeplitz operator $T_{Z^{\nu}}$, acting on $H^{2}(\Omega, \mu)$, where $\log \left(\Omega_{+}\right)$is the interior of the convex hull of the union of $\log (\operatorname{supp}(\lambda))$ and the cone $\widehat{-(\Gamma)}$.

(b) In view of Theorem 2.17, we now define first the Wiener-Hopf $C^{*}$-algebra on a pointed cone $\Gamma \supseteq \mathbf{Z}_{+}^{n}$ (i.e., $\Gamma \cap-\Gamma=\{0\}$ ) with a quasidecreasing weight function $\omega$ supported on $\Gamma$, as the $C^{*}$-algebra generated by the unweighted convolution operators $W_{\phi} g=\phi * g$, for $\phi \in l^{1}(\zeta)$, and $g \in l^{2}(\Gamma, \omega)$ (see the remarks following Lemma 2.3). This is then $*$-isomorphic to the Toeplitz $C^{*}$-algebra generated by $\left\{T_{Z^{\nu}}: \nu \in \Gamma\right\}$ acting on $H^{2}(\Omega, \mu)$, where $\Omega$ and $\mu$ are obtained from $\Gamma$ and $\omega$, according to part (a) of the above remark.

(c) Given a Reinhardt regular Borel positive finite measure $\mu$ and a pseudoconvex domain $\Omega$ such that $\operatorname{supp}(\mu) \subseteq \bar{\Omega} \subseteq \mathbf{C}^{n}$, let $\lambda$ be the positive part in the polar decomposition of $\mu$, i.e., $\mu=\lambda \times \theta$, where $\operatorname{supp}(\lambda) \subseteq \overline{\Omega_{+}}$. Also, for each $\nu \in \Gamma_{\Omega}$ (see (2)) let $d m_{\lambda}(\nu)$ be the regular Borel probability measure defined by

$$
d m_{\lambda}(\nu)=\frac{\left|r^{\nu}\right|^{2} d \lambda(r)}{\int\left|r^{\nu}\right|^{2} d \lambda(r)} .
$$

We will impose the condition on $\mu$ that the map $d m_{\lambda}$ from $\Gamma_{\Omega}$ to the space $\mathscr{P}\left(\overline{\Omega_{+}}\right)$of regular Borel probability measures on $\overline{\Omega_{+}}$(which is $w^{*}$-compact) can be extended continuously to the closure of $\Gamma_{\Omega}$ in an appropriate regular compactification of $\mathbf{R}^{n}$. This is the natural generalization of the compactification of $\mathbf{R}^{2}$ introduced earlier. We shall further ask that such a measure-valued map can be factored through a compact space defined in terms of $\partial \Omega$. These assumptions will allow us to produce the parameterized groupoid needed to study the Toeplitz $C^{*}$-algebra of part (b) of the present remark.

Definition 2.19. Let $Y$ be the set of all triples $(P, y, \mathscr{B})$, where $P$ is the orthogonal projection onto a subspace of $\mathbf{R}^{n}, y \in \operatorname{Ran}(P)$, and $\mathscr{B}$ is an 
ordered orthonormal basis for $\operatorname{Ran}(P)^{\perp}$. Given $x \in \mathbf{R}^{n}$, and an orthonormal set $\mathscr{A}=\left(u_{1}, \ldots, u_{m}\right)$, we say that $x$ is in an $\varepsilon$-neighborhood of $\mathscr{A}$ at infinity, and we write $x \in N_{\varepsilon}^{\infty}(\mathscr{A})$, when the following holds:

$$
\begin{gathered}
\|x\|>1 / \varepsilon, \quad\left\|u_{1}-\frac{x}{\|x\|}\right\|<\varepsilon, \\
\left\|x-\left\langle x, u_{1}\right\rangle u_{1}\right\|>1 / \varepsilon, \quad\left\|u_{2}-\frac{x-\left\langle x, u_{1}\right\rangle u_{1}}{\left\|x-\left\langle x, u_{1}\right\rangle u_{1}\right\|}\right\|<\varepsilon, \ldots, \\
\left\|x-\sum_{j=1}^{m-1}\left\langle x, u_{j}\right\rangle u_{j}\right\|>1 / \varepsilon, \quad\left\|u_{m}-\frac{x-\sum_{j=1}^{m-1}\left\langle x, u_{j}\right\rangle u_{j}}{\left\|\sum_{j=1}^{m-1}\left\langle x, u_{j}\right\rangle u_{j}\right\|}\right\|<\varepsilon .
\end{gathered}
$$

The topology of $Y$ is generated by the $\varepsilon$-neighborhoods of points $(P, y, \mathscr{B})$ of the form

$$
\begin{aligned}
&\{(Q, z, \mathscr{C}):\|y-P z\|<\varepsilon,\|P-Q P\|<\varepsilon, \\
& m=\operatorname{dim}(Q)-\operatorname{dim}(P), \mathscr{B}=\left(\mathscr{B}^{\prime}, \mathscr{B}^{\prime \prime}\right), \operatorname{Card}\left(\mathscr{B}^{\prime}\right)=m, \\
&\left.(1-P) z \in N_{\varepsilon}^{\infty}\left(\mathscr{B}^{\prime}\right),\left\|\mathscr{C}-\mathscr{B}^{\prime \prime}\right\|<\varepsilon\right\} .
\end{aligned}
$$

The space $Y$ will be called the flag compactification of $\mathbf{R}^{n}$. Given $\Omega$ and $\mu$ as in part (c) of the last remark, we let $Y_{+}$be the closure of $\Gamma_{\Omega}$ in $Y$. We henceforth shall consider the condition

(**) the map $d m_{\lambda}: \Gamma_{\Omega} \rightarrow \mathscr{P}\left(\overline{\Omega_{+}}\right)$of part (c) of the last remark can be extended to a continuous map $\widetilde{d m_{\lambda}}: Y_{+} \rightarrow \mathscr{P}\left(\overline{\Omega_{+}}\right)$.

As in the case $\Omega \subseteq \mathbf{C}^{2}$, and let $\mathscr{A}$ be the abelian $C^{*}$-subalgebra of $l^{\infty}\left(\mathbf{Z}^{n}\right)$ generated by $\operatorname{Tr}_{\mu} w_{\nu}$, where $w_{\nu}$ is given by

$$
\begin{aligned}
& w_{\nu}(\mu)=\frac{\left\|Z^{\nu+\mu}\right\|_{2, \lambda}}{\left\|Z^{\mu}\right\|_{2, \lambda}}, \quad \mu \in \Gamma_{\Omega}, \\
& w_{\nu}(\mu)=0, \quad \mu \notin \Gamma_{\Omega} .
\end{aligned}
$$

Let $X$ be the maximal ideal space of $\mathscr{A}$. Let $\tau, X_{+}$, and $\mathscr{G}$ be defined as in Definition 2.6, where we substitute $\mathbf{C}^{2}$ by $\mathbf{C}^{n}$ and $d m$ by $d m_{\lambda}$. Notice that in the definition of $\mathscr{G}=\left(X \times \mathbf{Z}^{n}\right)_{\mid X_{+}}$, we can let $X$ be the space generated by the $\mathbf{Z}^{n}$-orbits of $X_{+}$.

Theorem 2.20. Assume that $(* *)$ is satisfied. Then the map $\tau: \Gamma_{\Omega} \rightarrow X_{+}$is injective and has a (unique) continuous surjective covariant extension $\tilde{\tau}: Y_{+} \rightarrow X_{+}$. Furthermore, $C^{*}(\mathscr{G})$ is $*$-isomorphic to the $C^{*}$-algebra on $l^{2}\left(\Gamma_{\Omega}\right)$ generated by $\left\{\left(U_{\nu}, D_{\nu}\right): \nu \in \Gamma_{\Omega}\right\}$, as in Remark 2.10. Also, $\mathscr{K} \subseteq C^{*}(\mathscr{G})$, and

$$
C^{*}(\mathscr{G}) / \mathscr{K} \simeq C^{*}\left(\mathscr{G}_{\mid \partial X_{+}}\right),
$$

where $\partial X_{+}=X_{+} \backslash \tau\left(\Gamma_{\Omega}\right)$.

Proof. Let $(P, y, \mathscr{B}) \in Y_{+}$. For $\nu \in \Gamma_{\Omega}, \mu \in \mathbf{Z}^{n}$, and $y+P \mu \in \overline{P\left(\Gamma_{\Omega}\right)}$, we have:

$$
\left[(\tilde{\tau}(P, y, \mathscr{B}))\left(\operatorname{Tr}_{\mu} w_{\nu}\right)\right]^{2}=\int r^{2 \nu}\left(\widetilde{d m_{\lambda}}(P, y+P \mu, \mathscr{B})\right)(r) .
$$

It is easy to see that $\tilde{\tau}$ is continuous (because of $(* *)$ ). Also, $\tilde{\tau}=\tau$ is injective on $\Gamma_{\Omega}$. Indeed, assume that $\tau(\kappa)=\tau(l)$ for some $l, \kappa \in \Gamma_{\Omega}$. This means that 
if we let

$$
\begin{gathered}
\rho(\kappa, \mu, \nu)=\int r^{2 \nu}\left(\widetilde{d m_{\lambda}}(1, \mu+\kappa, \varnothing)\right)(r), \\
\nu, \kappa \in \Gamma_{\Omega}, \quad \mu \in \mathbf{Z}^{n}, \quad \kappa+\mu \in \Gamma_{\Omega}, \quad \rho(\kappa, \mu, \nu)=0, \quad \kappa+\mu \notin \Gamma_{\Omega},
\end{gathered}
$$

then we have that $\rho(\kappa, \mu, \nu)=\rho(l, \mu, \nu)$. We deduce that

$$
\kappa+m(\kappa-l) \in \Gamma_{\Omega}, \quad l+m(l-\kappa) \in \Gamma_{\Omega}
$$

for $m$ large enough. But, then

$$
\kappa-l \in \overline{\Gamma_{\Omega}^{\prime}} \cap-\overline{\Gamma_{\Omega}^{\prime}}=\{0\},
$$

as desired. An argument similar to the one given in Lemma 2.9 proves that $\tilde{\tau}$ is covariant and surjective. The rest of the statements are clear.

Definition 2.21. Let $\Omega$ be a normalized pseudoconvex Reinhardt domain, and let $\Gamma_{\Omega}=\left\{\nu \in \mathbf{Z}^{n}: Z^{\nu} \in H^{\infty}(\Omega)\right\}$. Let $\mathscr{F}(\Omega)$ be the set of all closed faces in the boundary of the closed solid convex set $\overline{\log \left(\Omega_{+}\right)}$(here, virtual faces are also included in $\mathscr{F}(\Omega)$, see $[19, \S 3])$. As before, we let $Y$ be the flag compactification of $\mathbf{R}^{n}$, and we let $Y_{+}$be the closure of $\Gamma_{\Omega}$ in $Y$. Let $\partial Y_{+}=$ $Y_{+} \backslash \Gamma_{\Omega}$, and let $Y(\Omega)$ be the set of all triples $(P, y+P \nu, \mathscr{B})$ in $Y$ such that $(P, y, \mathscr{B}) \in \partial Y_{+}$and $\nu \in \mathbf{Z}^{n}$. We also let $Z(\Omega)$ be the set of all pairs $(F, z)$, where $F \in \mathscr{F}(\Omega)$, and $z \in P_{[F]} \mathbf{Z}^{n}+\overline{P_{[F]} \Gamma_{\Omega}}$. Hereafter, $P_{[F]}$ denotes the (orthogonal) projection onto the linear manifold $[F]$ generated by $F$. We shall next define a map $q: Y(\Omega) \mapsto Z(\Omega)$. Let $\left(P, y+P_{[F]} \nu, \mathscr{B}\right) \in Y(\Omega)$, so that $(P, y, \mathscr{B}) \in \partial Y_{+}$and $\nu \in Z^{n}$. Let $\mathscr{B}=\left(u_{1}, \ldots, u_{m}\right)$ denote the corresponding orthonormal basis for $\operatorname{Ran}(P)^{\perp}$. We associate to $\mathscr{B}$ a flag of faces in $\mathscr{F}(\Omega)$ inductively as follows:

$$
F_{1}=\left\{y \in \overline{\log \left(\Omega_{+}\right)}:\left\langle y, u_{1}\right\rangle=\sup _{x \in \overline{\log \left(\Omega_{+}\right)}}\left\langle x, u_{1}\right\rangle\right\} .
$$

Having defined $F_{j}, 1 \leq j<m$, define

$$
F_{j+1}=\left\{y \in F_{j}:\left\langle y, u_{j+1}\right\rangle=\sup _{x \in F_{j}}\left\langle x, u_{j+1}\right\rangle\right\} .
$$

(Notice that $\left[F_{m}\right]$ is a translate of $\operatorname{Ran}(P)$.) We then define $q(P, y+P \nu, \mathscr{B})=$ $\left(F_{m}, P_{\left[F_{m}\right]}(y+\nu)\right)$. We provide $Z(\Omega)$ with the quotient topology (note that $q$ is surjective). We denote additively the natural action of $Z^{n}$ on $Y(\Omega)$ and $Z(\Omega)$. Let $Z_{+}=q\left(\partial Y_{+}\right)$.

Lemma 2.22. The map $q: Y(\Omega) \mapsto Z(\Omega)$ is covariant and induces a groupoid $C^{*}$-algebra monomorphism

$$
q_{*}: C^{*}\left[\left(Y(\Omega) \times \mathbf{Z}^{n}\right)_{\mid \partial Y_{+}}\right] \mapsto C^{*}\left[\left(Z(\Omega) \times \mathbf{Z}^{n}\right)_{\mid Z_{+}}\right] .
$$

Proof. We first observe that the closure of $-\widehat{\log \left(\Omega_{+}\right)}$in $Y \backslash \mathbf{R}^{n}$ coincides with $\overline{\Gamma_{\Omega}} \backslash \mathbf{R}^{n}$. Since $q$ is obviously surjective (as observed above) and commutes with the action of $Z^{n}$, it induces an injective *-homomorphism at the $C^{*}$-algebra level. Further, since $q^{-1}\left(Z_{+}\right)=\partial Y_{+}, q$ induces a $*$-monomorphism between the corresponding reduced groupoid $C^{*}$-algebras. 
Definition 2.23. Given a Reinhardt regular Borel positive finite measure $\mu$ supported in the closure of a bounded pseudoconvex Reinhardt domain $\Omega$, as above, we say that $\mu$ is well behaved if condition (**) holds, and the following condition is satisfied:

$(* * *)$

The map $\widetilde{d m_{\lambda}}: \partial Y_{+} \mapsto \mathscr{P}(\partial \Omega)$ factors through $Z^{+}$to a continuous map $\widehat{d m_{\lambda}}: Z_{+} \mapsto \mathscr{P}(\partial \Omega)$, via the map $q: \partial Y_{+} \mapsto Z_{+}$, so that $\operatorname{supp}\left({\widehat{d m_{\lambda}}}_{\lambda}(F, z)\right) \subseteq \overline{\operatorname{Exp}(F)}$, for every $(F, z) \in Z_{+}$.

Theorem 2.24. Let $\Omega, \mu$ be as above, and assume that $\mu$ is well behaved and that $\Omega$ is normalized and satisfies condition $(*)$, i.e., $\mathbf{Z}^{n} \cap-\overline{\log \left(\Omega_{+}\right)}=\Gamma_{\Omega}$. Then, via $q, \tilde{\tau}$ induces a covariant homeomorphism $\hat{\tau}: Z(\Omega) \mapsto \partial X$, where $\partial X=X \backslash \tau\left(\mathbf{Z}^{n}\right)$, so that $C^{*}\left(\partial X \times \mathbf{Z}^{n}\right)$ is *-isomorphic to $C^{*}\left(Z(\Omega) \times \mathbf{Z}^{n}\right)$ : In fact, the map $\hat{\tau}$ is given by

$$
\begin{gathered}
(\hat{\tau}(F, z))\left(\operatorname{Tr}_{\mu} w_{\nu}\right)=\int r^{2 \nu}\left(\widehat{d m_{\lambda}}\left(F, z+P_{[F]} \mu\right)\right)(r) . \\
\nu \in \Gamma_{\Omega}, \quad z+P_{[F]} \mu \in P_{[F]} \overline{\Gamma_{\Omega}}, \\
(\hat{\tau}(F, z))\left(\operatorname{Tr}_{\mu} w_{\nu}\right)=0, \quad \nu \in \Gamma_{\Omega}, \quad z+P_{[F]} \mu \in[F] \backslash \overline{P_{[F]} \Gamma_{\Omega}} .
\end{gathered}
$$

Furthermore, $\hat{\tau}$ maps homeomorphically and covariantly $Z_{+}$onto $\partial X_{+}$, so that $C^{*}(\mathscr{G}) / \mathscr{K} \simeq C^{*}(\mathscr{G}(\Omega))$, where $\mathscr{G}(\Omega)$ is the groupoid defined by $\mathscr{G}(\Omega)=$ $\left(Z(\Omega) \times \mathbf{Z}^{n}\right)_{\mid Z_{+}}$.

Proof. The proof that $\hat{\tau}$ is surjective is similar to that of Lemma 2.9. Indeed, let $x \in \partial X$. Then there exists $\left\{\nu_{k}\right\} \subseteq \Gamma_{\Omega}$ and $\mu \in \mathbf{Z}^{n}$ such that $\tau\left(\mu+\nu_{k}\right) \rightarrow x$. Without loss of generality, we can assume that $\left\|\nu_{k}\right\| \rightarrow \infty$. Thus, we can drop to a subsequence, and suppose that $\nu_{k} \rightarrow(P, y, \mathscr{B}) \in \partial Y_{+}$. Let $(F, z)=$ $q(P, y, \mathscr{B})$. It follows that

$$
x=\lim \tau\left(\mu+\nu_{k}\right)=\tilde{\tau}(P, y+P \mu, \mathscr{B})=\hat{\tau}\left(F, z+P_{[F]} \mu\right) .
$$

In order to prove injectivity, let $(F, z),(G, w) \in Z(\Omega)$, such that $\hat{\tau}(F, z)=$ $\hat{\tau}(G, w)$. We claim that $F=G$. Since $\Omega$ satisfies condition $(*)$, and ${\widehat{d m_{\lambda}}}_{\lambda}(F, z)-{\widehat{d m_{\lambda}}}_{\lambda}(G, w)$ is orthogonal to $R^{2 \nu}, \forall \nu \in \Gamma_{\Omega}$, we deduce that $d m^{\prime}=\widehat{d m}_{\lambda}(F, z)={\widehat{d m_{\lambda}}}_{\lambda}(G, w)$. By condition $(* * *), \operatorname{supp}\left(d m^{\prime}\right) \subseteq F \cap G$. For each $\nu \in \Gamma_{\Omega}$, let $f_{\nu}: \mathbf{Z}^{n} \mapsto \mathbf{R}_{+}$and $g_{\nu}: \mathbf{Z}^{n} \mapsto \mathbf{R}_{+}$, be defined by

$$
\begin{array}{rc}
f_{\nu}(\mu)=\int r^{2 \nu}\left(\widehat{d m_{\lambda}}\left(F, z+P_{[F]} \mu\right)\right)(r), & z+P_{[F]} \mu \in \overline{P_{[F]} \Gamma_{\Omega}}, \\
f_{\nu}(\mu)=0, & z+P_{[F]} \mu \notin \overline{P_{[F]} \Gamma_{\Omega}}, \\
g_{\nu}(\mu)=\int r^{2 \nu}\left(\widehat{d m_{\lambda}}\left(G, w+P_{[G]} \mu\right)\right)(r), & w+P_{[G]} \mu \in \overline{P_{[G]} \Gamma_{\Omega}}, \\
g_{\nu}(\mu)=0, & w+P_{[G]} \mu \notin \overline{P_{[G]} \Gamma_{\Omega}} .
\end{array}
$$

Our assumption that $\hat{\tau}(F, z)=\hat{\tau}(G, w)$ is equivalent to $f_{\nu}=g_{\nu}, \forall \nu \in \Gamma_{\Omega}$. In particular, for every $\nu \in \Gamma_{\Omega}$, we have

$$
-z+\overline{P_{[F]} \Gamma_{\Omega}}=\operatorname{supp}\left(f_{\nu}\right)=\operatorname{supp}\left(g_{\nu}\right)=-w+\overline{P_{[G]} \Gamma_{\Omega}} .
$$

This implies that $F=G$, as desired. Let $\left\{\nu_{k}\right\},\left\{\mu_{k}\right\}$ be sequences in $\mathbf{Z}^{n}$ such that $P_{[F]} \nu_{k} \rightarrow(F, z)$ and $P_{[F]} \mu_{k} \rightarrow(F, w)$. By continuity, we then have: 


$$
\begin{aligned}
\hat{\tau}(F, z+(z-w)) & =\lim \hat{\tau}\left(F, z+P_{[F]}\left(\nu_{k}-\mu_{k}\right)\right) \\
& =\lim \hat{\tau}\left(F, w+P_{[F]}\left(\nu_{k}-\mu_{k}\right)\right) \\
& =\hat{\tau}(F, w+(z-w))=\hat{\tau}(F, z) .
\end{aligned}
$$

Therefore, we also have $\hat{\tau}(F, z+m(z-w))=\hat{\tau}(F, z)$, for every $m \in \mathbf{Z}_{+}$. If $z-w \neq 0$, we can find a strictly increasing sequence $\left\{m_{k}\right\} \subseteq \mathbf{Z}_{+}$such that $\left(F, z+m_{k}(z-w)\right) \rightarrow(H, y) \in Z_{+}$, so that $\operatorname{dim}(H)<\operatorname{dim}(\bar{F})$. Taking limits again, we see that $\hat{\tau}(F, z)=\hat{\tau}(H, y)$. Reasoning as above, we then have $F=H$ which is impossible. Therefore, we proved that $\hat{\tau}$ is injective. The rest of the statements are clear.

Remark 2.25. (a) The simplest candidate for a well behaved Reinhardt measure $\mu=\lambda \times \theta$ on a domain $\Omega$ that satisfies condition $(*)$ is obtained by letting $\lambda$ be the Dirac mass at $1 \in \Omega_{+}$, where $\log \left(\Omega_{+}\right)=-\widehat{\Gamma}$ and $\Gamma \subseteq \mathbf{Z}^{n}$ is a pointed cone containing $\mathbf{Z}_{+}^{n}$. Following [12], we say that a closed solid convex set $C \subseteq \mathbf{R}^{n}$ is tamed, if the map $\gamma:[0,1] \times C \times C$ defined by $\gamma(\lambda, x, y)=\lambda x+(1-\lambda) y$, $\lambda \in[0,1], x, y \in C$ is open. This is equivalent to the following condition: if $z=\lambda x+(1-\lambda) y \in C$, and $\lambda \in[0,1], x, y \in C$, and $\left\{z_{k}\right\}$ is a sequence in $C$ that tends to $z$, then there exists $k_{0} \in \mathbf{Z}_{+}$such that for every $k>k_{0}$ there are $\lambda_{k} \in[0,1], x_{k}, y_{k} \in C$ with $z_{k}=\lambda_{k} x_{k}+\left(1-\lambda_{k}\right) y_{k}$ and such that $\lambda_{k} \rightarrow \lambda$, $y_{k} \rightarrow y, x_{k} \rightarrow x$. From the results of $\S \S 4,5$, and 6 of [12], it follows that if $\Omega$ and $\mu$ are as in the present remark, and if $\log \left(\Omega_{+}\right)$is tamed, then $\mu$ is well behaved.

(b) Other good candidates for a well-behaved measure are obtained by letting $\mu$ be either the volume Lebesgue measure on $\Omega$ or the surface Lebesgue measure on $\partial \Omega$ (this is the significance of the results in [22]). It is not hard to prove that if $\mathscr{F}(\Omega) \backslash \mathscr{F}(\Omega)$ is finite then $\mu$ is well behaved (we point out that these kind of domains are tamed). As observed in [12], closed solid convex subsets in $\mathbf{R}^{2}$ are always tame, so if $\Omega$ is a normalized Reinhardt domain in $\mathbf{C}^{2}$, then $\log \left(\Omega_{+}\right)$is tamed; the results of $\S 2$ show precisely that the Lebesgue measure on $\Omega$ is well behaved. In [20], more general examples of well-behaved measures are presented.

(c) From Theorem 2.24, we see that the study of $C^{*}\left(T_{Z}\right)$ acting on $H^{2}(\Omega, \mu)$, where $\mu$ is well behaved supported on $\bar{\Omega}$, and $\Omega$ is a (bounded) normalized pseudoconvex Reinhardt domain satisfying condition $(*)$, is reduced to the study of $C^{*}(\mathscr{G}(\Omega))$, which is a purely geometrical object. Letting $\mathscr{G}_{k}(\Omega)$ be the groupoid obtained by reducing $\mathscr{G}(\Omega)$ to $\mathscr{F}_{k}(\Omega), 0 \leq k<n$, it is easy to see that $C^{*}(\mathscr{G}(\Omega))$ has a decomposition series of length $n$, where the quotient of the $k$ th ideal by the $k-1$ th ideal is *-isomorphic to $C^{*}\left(\mathscr{G}_{k}(\Omega)\right)$. On the other hand, $C^{*}\left(\mathscr{G}_{k}(\Omega)\right)$ can be analyzed in terms of foliation $C^{*}$-algebras associated with the open $k$ th dimensional faces of $\partial \log \left(\Omega_{+}\right)$, just as in the case $n=2$ discussed above.

(d) Using parts (a) and (b) above, we can give a rather elegant positive solution of the index problem presented in [13] for the general case of the (unweighted) Wiener-Hopf $C^{*}$-algebra over a pointed polyhedral cone $\Gamma$, such that $\mathbf{Z}_{+}^{N} \subseteq \Gamma \subseteq \mathbf{Z}^{N}$. Indeed, let $\Omega$ be the (pseudoconvex) Reinhardt domain defined by the condition $\log \left(\Omega_{+}\right)=-\widehat{\Gamma}$. Then, by [19, Theorem 1.3], given any point $w \in \Omega$, the $n$-tuple $T_{Z}-w$ is Fredholm and has index $(-1)^{n}$. This is equivalent to the assertion that the $2^{n-1} \times 2^{n-1}$ operator matrix $\widehat{T_{Z}-w}$ associated 
with $T_{Z}-w$ according to [2] has the same index. For example, for $n=2$, $\widehat{T_{Z}-w}$ is the $2 \times 2$ matrix

$$
\left(\begin{array}{cc}
T_{Z_{1}}-w_{1} & T_{Z_{2}}-w_{2} \\
-\left(T_{Z_{2}}-w_{2}\right)^{*} & \left(T_{Z_{1}}-w_{1}\right)^{*}
\end{array}\right)
$$

Since $C^{*}\left(T_{Z}\right)$ is unitarily equivalent to a subalgebra of $\mathscr{W}(\Gamma, \omega)$, where $\omega$ is the weight function induced by the Lebesgue measure on $\Omega$, and the weighted and unweighted Wiener-Hopf $C^{*}$-algebras are $*$-isomorphic (by $(B)$ ), the $K$ theoretical index problem posed in [13] has an affirmative answer in this case.

\section{Tube Domains}

We shall first introduce the notion of weighted Wiener-Hopf operators associated to a pointed solid cone $\Gamma \subseteq \mathbf{R}^{n}$ and a quasidecreasing function $\omega$ supported on $\Gamma$. We shall base our definition on the model found in the previous section where the group in question was $\mathbf{Z}^{n}$.

Definition 3.1. A closed convex cone $\Gamma \subseteq \mathbf{R}^{n}$ is called solid if $\overline{(\Gamma 0)}=\Gamma$. A Borel measurable function $\omega: \mathbf{R}^{n} \rightarrow \mathbf{R}_{+}$is called quasidecreasing (with respect to $\Gamma$ ) if $\omega$ is supported on $\Gamma$, it is strictly positive on $\Gamma$ and

$$
\zeta^{\prime}(s)=\sup _{t \in \Gamma} \frac{\omega(s+t)}{\omega(t)}<\infty, \quad \forall s \in \Gamma .
$$

Let

$$
\zeta(s)=\max \left(\zeta^{\prime}(s), 1\right), \quad s \in \Gamma, \quad \zeta(s)=0, \quad s \notin \Gamma .
$$

(Observe that $\omega$ is decreasing if and only if $\zeta=\chi_{\Gamma}$.) We let $L^{2}(\Gamma, \omega)$ be the space of all those measurable functions $g$ supported on $\Gamma$, and such that $\int|g(t)|^{2} \omega^{2}(t) d \eta(t)<\infty$, where $\eta$ is the Lebesgue measure on $\mathbf{R}^{n}$. Given $\phi \in L^{1}(\Gamma, \zeta)$, we define the Wiener-Hopf operator $W_{\phi}$ with symbol $\phi$ by

$$
\left(W_{\phi} g\right)(t)=(\phi * g)(t) \text {. }
$$

Remark 3.2. (a) Just as we saw in the preceding section, $L^{1}(\Gamma, \zeta)$ (which is defined in the obvious manner) is a commutative Banach algebra, and the inclusion $L^{1}(\Gamma, \zeta) \mapsto L^{1}\left(\Gamma, \chi_{\Gamma}\right) \quad\left(=L^{1}(\Gamma)\right)$ is norm decreasing. From the fact that $\omega$ is quasidecreasing, it easily follows that $W_{\phi}$ is a bounded operator on $L^{2}(\Gamma, \omega)$ such that $\left\|W_{\phi}\right\| \leq\|\phi\|_{1, \zeta}$. We denote by $\mathscr{W}(\Gamma, \omega)$ the $C^{*}$-algebra generated by $W_{\phi}$, with $\phi$ running in $L^{1}(\Gamma, \zeta)$.

(b) Let $L^{2}(\Gamma)$ be the subspace of $L^{2}\left(\mathbf{R}^{n}, \eta\right)$ of those functions supported in $\Gamma$. Let $U: L^{2}(\Gamma, \omega) \mapsto L^{2}(\Gamma)$ be the unitary transformation defined by $U g=\omega g$. Given $\phi \in L^{1}(\Gamma, \zeta)$, we then have

$$
\left[\left(U W_{\phi} U^{*}\right) g\right](t)=\int_{\Gamma} \phi(s) \frac{\omega(t) \chi_{\Gamma}(t-s)}{\omega(t-s)} g(t-s) d \eta(s) .
$$

Thus, $\mathscr{W}(\Gamma, \omega)$ is unitarily equivalent to the $C^{*}$-algebra generated by integral operators whose kernels are functions of the form

$$
\phi(t-s) \frac{\omega(t) \chi_{\Gamma}(s)}{\omega(s)}, \quad s, t \in \mathbf{R}^{n}
$$

(c) As in the case of $\mathbf{Z}^{n}$, there is a natural way of producing pairs $(\Gamma, \omega)$ of the above kind (see below). But first we need to deal with the title of the present 
section. We recall that $\Omega \subseteq \mathbf{C}^{n}$ is called a tube domain if it is a domain of the form $\Omega=\left\{z \in \mathbf{C}^{n}: \operatorname{Re}(z) \in C\right\}$, where $C \subseteq \mathbf{R}^{n}$. By [7], $\Omega$ is pseudoconvex if and only if $C=\operatorname{Re}(\Omega)$ is convex. The image of a tube domain $\Omega$ under the exponential map $\operatorname{Exp}(\Omega)$ is a Reinhardt domain. Of course, $\operatorname{Exp}(\Omega)$ is pseudoconvex if and only if $\Omega$ is pseudoconvex.

Definition 3.3. Let $\Omega$ be a tube domain such that $\operatorname{Exp}(-\Omega)$ is bounded. A tube measure $\mu$ on $\Omega$ is a measure of the form $\mu=\lambda \times \eta$, where $\eta$ is the Lebesgue measure on $i \mathbf{R}^{n}$, and $\lambda$ is a regular Borel positive finite measure supported on $\overline{\operatorname{Re}(\Omega)}$. Let $H^{2}(\Omega, \mu)$ be the closure in $L^{2}(\Omega, \mu)$ of $H^{\infty}(\Omega) \cap L^{2}(\Omega, \mu)$. Let $\Gamma \subseteq \mathbf{R}^{n}$ be the (convex) cone $\left\{x \in \mathbf{R}^{n}: \operatorname{Exp}(-\langle x,\rangle.) \in L^{\infty}(\Omega)\right\}$ and let $\omega: \mathbf{R}^{n} \rightarrow \mathbf{R}_{+}$be defined by

$$
\omega^{2}(t)=\int e^{-2\langle t, s\rangle} d \lambda(s), \quad t \in \Gamma, \quad \omega(t)=0, \quad t \notin \Gamma,
$$

so that $\operatorname{supp}(\omega) \subseteq \Gamma$.

Notice that we can substitute $\operatorname{Re}(\Omega)$ in the above definition by

$$
\operatorname{conv}(\operatorname{Re}(\Omega) \cup \widehat{\Gamma}) \text {. }
$$

The following lemma establishes the connection between Toeplitz operators on $H^{2}(\Omega, \mu)$ and Wiener-Hopf operators on $L^{2}(\Gamma, \omega)$. It is somewhat standard, but since we could not find it in its present form within the existing literature, we include it here for completeness.

Lemma 3.4. The Laplace transform $L$ defines an isometry from $L^{2}(\Gamma, \omega)$ into $L^{2}(\Omega, \mu)$ whose range is $H^{2}(\Omega, \mu)$ i.e., the closure of $L^{2}(\Omega, \mu) \cap H^{\infty}(\Omega)$. Furthermore, $L \phi \in H^{\infty}(\Omega), \forall \phi \in L^{1}(\Gamma, \zeta)$, and $L W_{\phi} L^{*}=T_{L \phi}$, where $T_{\psi}$ denotes the Toeplitz operator with symbol $\psi \in L^{\infty}(\Omega)$ on $H^{2}(\Omega, \mu)$.

Proof. Since $C_{c}(\Gamma)$ is dense in $L^{2}(\Gamma, \omega)$, in order to prove that $L$ is isometric it is enough to check it for $g \in C_{c}(\Gamma)$. Note that $L g \in H^{\infty}(\Omega)$, so that $\operatorname{Ran}(L) \subseteq H^{2}(\Omega, \mu)$. Given $f \in L^{1}(\Gamma, \zeta), x \in \operatorname{Re}(\Omega)$, let $f_{x}(t)=e^{-\langle x, t\rangle} f(t)$. Also, let $\tilde{f}$ denote the Fourier transform of $f$. Using Parseval's identity, we have

$$
\begin{aligned}
\|L g\|^{2} & =\int_{\Omega}\left|\widetilde{g_{x}}(y)\right|^{2} d \mu(x, y)=\int_{\operatorname{Re}(\Omega)}\left(\int_{\mathbf{R}^{n}}\left|g_{x}(t)\right|^{2} d \eta(t)\right) d \lambda(x) \\
& =\int_{\Gamma}|g(t)|^{2}\left[\int_{\operatorname{Re}(\Omega)} e^{-2\langle x, t\rangle} d \lambda(x)\right] d \eta(t) \\
& =\int_{\Gamma}|g(t)|^{2} \omega^{2}(t) d \eta(t)=\|g\|_{2, \omega}^{2} .
\end{aligned}
$$

By [8, Theorem 7.4.3], $\operatorname{Ran}(L)=H^{2}(\Omega, \mu)$. If $\phi \in L^{1}(\Gamma, \zeta)$, then

$$
\begin{aligned}
|(L \phi)(x+i y)| & \leq \int_{\Gamma}|\phi(t)| e^{-\langle x, t\rangle} d \eta(t) \\
& \leq\|\phi\|_{1, \zeta}, \quad \forall(x+i y) \in \Omega .
\end{aligned}
$$

Remark 3.5. It is natural to ask under what conditions, given a (quasidecreasing) weight function $\omega: \Gamma \mapsto \mathbf{R}_{+}$, there exists a regular Borel positive finite measure 
$\lambda$ such that, for some $x \in \mathbf{R}^{n}$, we have

$$
\omega^{2}(t)=\int_{x+\widehat{\Gamma}} e^{-2\langle t, s\rangle} d \lambda(s), \quad t \in \Gamma .
$$

There are various necessary conditions for the validity of (24). First, observe that if (24) holds, then, $\forall t \in[0,1]^{n} \cap \Gamma$, we have

$$
\omega^{2}(t+\nu)=\int_{x+\widehat{\Gamma}} e^{-2\langle t+\nu, s\rangle} d \lambda(s), \quad \forall \nu \in \mathbf{Z}^{n} \cap \Gamma .
$$

Also, notice that we already know a criterion for the existence of a measure $\lambda$ that satisfies (25) for $t=0$, when $\Gamma \supseteq \mathbf{R}_{+}^{n}$. In fact, by Theorem 2.17, such a measure $\lambda$ exists if and only if $\left\{\omega(\nu), \nu \in \Gamma \cup \mathbf{Z}^{n}\right\}$ gives rise to a weighted $l^{2}$-space $l^{2}(\omega)$ on which the semigroup $W_{\nu}: \nu \in \Gamma$ of forward (unweighted) shifts is subnormal. Furthermore, such a $\lambda$ is uniquely determined. Thus, to answer the above question we may use Theorem 2.17, together with formula (25) for $t \in(0,1)^{n} \cap \Gamma$. Another criterion for the existence of $\lambda$ is obtained by employing the fact that $\omega$ must be extendable to a holomorphic function on the tube domain $\left\{z \in \mathbf{C}^{n}: \operatorname{Re}(z) \in x+\Gamma^{0}\right\}$, and then use [8, Theorem 7.4.2].

Definition 3.6. Throughout the rest of this section, we shall assume that $\mu=$ $\lambda \times \eta$ is a tube measure according to Definition 3.3, and that $\Gamma$ and $\omega$ are defined as in Definition 3.3. We shall also suppose that $\Omega$ is pseudoconvex. Notice that the condition " $\operatorname{Exp}(-\Omega)$ bounded" implies that

$$
\Gamma=\bigcup_{x \in \mathbf{R}^{n}} x+\widehat{\operatorname{Re}(\Omega)} \text {. }
$$

Just as in the previous section and in view of (23), we let $\mathscr{A}$ be the (abelian) $C^{*}$-subalgebra of $L^{\infty}\left(\mathbf{R}^{n}\right)$ generated by translates of functions of the form

$$
f_{r, \phi}(t)=\int_{\Gamma} \phi(s) w_{r}(t-s) d \eta(s), \quad t \in \mathbf{R}^{n}, \quad r \in \Gamma,
$$

where

$$
w_{r}(s)=\frac{\omega(r) \chi_{\Gamma}(s)}{\omega(s)}, \quad s \in \mathbf{R}^{n}, \quad r \in \Gamma .
$$

Observe that the functions $f_{r, \phi}$ are supported on $\Gamma$. Also, we let $X$ be the maximal ideal space of $\mathscr{A}$, and we let $\tau: \mathbf{R}^{n} \mapsto X$, be given by

$$
\begin{aligned}
{[\tau(t)]\left(\operatorname{Tr}_{u} f_{r, \phi}\right) } & =\left(\operatorname{Tr}_{u} f_{r, \phi}\right)(t) \\
& =\int_{\Gamma} \phi(s) \frac{\omega(r) \chi_{\Gamma}(t+u-s)}{\omega(t+u-s)} d \eta(s), \quad t, u \in \mathbf{R}^{n}, \quad r \in \Gamma .
\end{aligned}
$$

It follows that $\mathbf{R}^{n}$ acts continuously on $X$. We let $X_{+}=\overline{\tau(\Gamma)}$, and we let $\mathscr{G}$ be the groupoid $\left(X \times \mathbf{R}^{n}\right)_{\mid X_{+}}$. We conjecture $C^{*}(\mathscr{G})\left(=C_{\mathrm{red}}^{*}(\mathscr{G})\right)$ is of type $\mathrm{I}$.

\section{REINHARDT-TUBE DOMAINS}

The above discussion leads naturally to the following more general definition (we point out the unimodularity assumption is not essential and it is introduced here in order to avoid notational complications). 
Definition 4.1. Let $G$ be a second countable unimodular locally compact Hausdorff group, and let $\Gamma$ be a pointed solid cone of $G$ (i.e., a normal subsemigroup such that $\overline{\left(\Gamma^{0}\right)}=\Gamma, \Gamma \cap \Gamma^{-1}=\{e\}$ where $e$ is the identity of $G$, and such that $\Gamma$ generates $G$ ). Let $\omega: G \mapsto \mathbf{R}_{+}$be a quasidecreasing Borel function supported on $\Gamma$, i.e., $\omega$ is strictly positive on $\Gamma$, and

$$
\zeta^{\prime}(s)=\sup _{t s^{-1} \in \Gamma} \frac{\omega(t) \chi_{\Gamma}(s)}{\omega\left(t s^{-1}\right)}<\infty .
$$

Also, let $\lambda$ be a fixed left Haar measure on $G$, and let $L^{1}(\Gamma, \zeta)=L^{1}(G, \zeta d \lambda)$, where $\zeta=\max \left(\zeta^{\prime}, \chi_{\Gamma}\right)$. Given $\phi \in L^{1}(\Gamma, \zeta)$, let $W_{\phi}$ be the Wiener-Hopf operator on $L^{2}(\Gamma, \omega)=L^{2}\left(G, \omega^{2} d \lambda\right)$ with symbol $\phi$, namely

$$
\left(W_{\phi} g\right)(t)=(\phi * g)(t)=\int \phi(s) g\left(t s^{-1}\right) d \lambda(s), \quad t \in \Gamma, \quad g \in L^{2}(\Gamma, \omega) .
$$

The Wiener-Hopf $C^{*}$-algebra $\mathscr{W}(\Gamma, \omega)$ is the $C^{*}$-algebra generated by $\left\{W_{\phi}\right.$ : $\left.\phi \in L^{1}(\Gamma, \zeta)\right\}$.

Remark 4.2. (a) If $\omega=1$ we obtain the standard Wiener-Hopf $C^{*}$-algebra on $\Gamma$ first introduced in [11]. As it is customary by now, let $X$ be the spectrum of the abelian $C^{*}$-subalgebra of $L^{\infty}(G)$ generated by right translates of functions of the form:

$$
\begin{aligned}
f_{r, \phi}(t)=\left(\phi * w_{r}\right)(t)=\int \phi(s) w_{r}\left(t s^{-1}\right) & d \lambda(s), \\
& \phi \in L^{1}(\Gamma, \zeta), \quad r \in \Gamma, \quad t \in G,
\end{aligned}
$$

where $w_{r}$ is the bounded Borel function defined, for $r \in \Gamma$, by

$$
w_{r}(s)=\frac{\omega(r) \chi_{\Gamma}\left(r s^{-1}\right)}{\omega\left(r s^{-1}\right)}, \quad s \in G .
$$

Then $G$ acts on $X$ via a natural map $(x, t) \rightarrow x t$. Further, evaluation at points of $G$ yields a mapping $\tau: G \mapsto X$ whose range is dense in $X$. Let $X_{+}=\overline{\operatorname{Ran}(\tau)}$, and let $\mathscr{G}$ be the groupoid

$$
\mathscr{G}=(X \times G)_{\mid X_{+}}=\left\{(x, t) \in X \times G: x \in X_{+}, x t \in X_{+}\right\},
$$

i.e., $\mathscr{G}$ is the reduction of the transformation group $X \times G$ to $X_{+}$. Under mild assumptions, we can sometimes identify $\mathscr{W}(\Gamma, \omega)$ as a natural subalgebra of $C_{\text {red }}^{*}(\mathscr{G})$. Also, a natural decomposition series of $C_{\text {red }}^{*}(\mathscr{G})$ can be computed in terms of groupoid $C^{*}$-algebras associated with the dual faces of $\Gamma$.

(b) As we have seen in the previous two sections, the general strategy to parameterize $\mathscr{G}$ is to assume that $\omega$ is the Laplace transform of a regular Borel positive finite measure $\lambda$ supported on a dual convex set $C$. One then embeds $\Gamma$ in a suitable Hausdorff compactification $Y$ of $G$ and imposes the condition that the map $d m_{\lambda}: \Gamma \mapsto \mathscr{P}(\bar{C})$ induced by the normalized momenta of $\lambda$ be extendable to a map $\widetilde{d m_{\lambda}}$ on the closure $Y_{+}$of $\Gamma$ in $Y$. One then defines a surjection $q: \partial Y_{+}=Y_{+} \backslash \Gamma \rightarrow Z_{+}$where $Z_{+}$is constructed employing the boundary geometry of $C$. One then further requires that $\widetilde{d m_{\lambda}}$ factors out through a continuous map $\widetilde{d m_{\lambda}}: Z_{+} \mapsto \partial X_{+}=X_{+} \backslash \tau(\Gamma)$. These assumptions allow one to prove that $C_{\text {red }}^{*}(\mathscr{G})$ is $*$-isomorphic to the reduced $C^{*}$-algebra of a groupoid associated with $Z_{+}$, which is a purely geometrical object. We give another illustration of this strategy, by considering the dual of a connected abelian locally compact Lie group, i.e., a group of the form $G=\mathbf{R}^{n} \times \mathbf{T}^{m}$. 
Definition 4.3. We say that a domain $\Omega \subseteq \mathbf{C}^{m+n}$ is a Reinhardt-tube domain if it is the inverse image of a Reinhardt domain under the map Id $\times$ Exp defined by

$$
\operatorname{Id} \times \operatorname{Exp}(z, w)=(z, \operatorname{Exp}(w)), \quad z \in \mathbf{C}^{n}, \quad w \in \mathbf{C}^{m} .
$$

Remark 4.4. (a) It readily follows, from [7, Theorem 2.5.7], that a Reinhardttube domain $\Omega \subseteq \mathbf{C}^{m+n}$ is pseudoconvex if and only if

$$
\log \times \operatorname{Re}(\Omega)=\left\{(x, y) \in \mathbf{R}^{m+n}:(\operatorname{Exp}(x), y) \in \Omega\right\}
$$

is convex.

(b) Here is the simplest example of a nontrivial pseudoconvex Reinhardttube domain. Let $0 \leq \alpha<\beta<\infty$, and let $\Omega=(\operatorname{Exp} \times \mathrm{Id})^{-1}\left(\Omega_{\alpha, \beta}\right)$, where $\Omega_{\alpha, \beta}$ is the generalized Hartogs wedge considered in $\S 2$. Alternatively,

$$
\mathbf{\Omega}=\left\{(z, w) \in \mathbf{C}^{2}:|z|^{\alpha}<|\operatorname{Exp}(w)|<|z|^{\beta},|z|<1\right\} .
$$

Let $\lambda$ be the point mass measure at $(1,0) \in \Omega$. We consider the Toeplitz $C^{*}$ algebra $\mathscr{T}(\Omega)$ generated by $T_{Z}$ and $T_{\operatorname{Exp}(W)}$. Using the techniques suggested in Remark 4.2, it follows that $\mathscr{T}(\Omega)$ is of type I. In fact, if $\mathscr{I}$ denotes the commutator ideal of $\mathscr{T}(\Omega)$, then

$$
\begin{aligned}
& \mathscr{K} \subseteq \mathscr{I}, \quad \mathscr{T}(\Omega) / \mathscr{I} \simeq C_{0}(\mathbf{T} \times \mathbf{R}), \\
& \mathscr{I} / \mathscr{K} \simeq(C(\mathbf{T} \otimes \mathscr{K})) \oplus\left(\mathscr{K} \otimes C_{0}(\mathbf{R})\right) .
\end{aligned}
$$

(c) Employing an analysis similar to that of the previous two sections, one can introduce the notion of a Reinhardt-tube measure $\mu$ on a pseudoconvex Reinhardt-tube domain $\Omega \subseteq \mathbf{C}^{m+n}$. One then defines the cone $\Gamma_{\Omega}$ by

$$
\Gamma_{\Omega}=\left\{(k, s) \in \mathbf{Z}^{m} \times \mathbf{R}^{n}:\left|Z^{k}\right|\left|e^{\langle s, W\rangle}\right| \in L^{\infty}(\Omega)\right\}
$$

and considers the weight function $\omega$ supported on $\Gamma_{\Omega}$ and defined by

$$
\omega(k, t)=\int\left|z^{k}\right|^{2} e^{2\langle t, w\rangle} d \mu(z, w), \quad(k, t) \in \Gamma_{\Omega} .
$$

One can then study the weighted Wiener-Hopf $C^{*}$-algebra $\mathscr{W}\left(\Gamma_{\Omega}, \omega\right)$ as was done in the previous two sections, via the boundary geometry of $\Omega$. When $\log \times \operatorname{Re}(\Omega)$ is a tamed convex set, one can produce examples of well behaved Reinhardt-tube measures using, for instance, a suitable normalization of the Lebesgue measure on $\Omega$. The resulting classification of $\mathscr{W}\left(\Gamma_{\Omega}, \omega\right)$ generalizes the results of $[4,11,19]$.

\section{REFERENCES}

1. E. Bedford and J. Dadok, Generalized Reinhardt domains, preprint, 1990.

2. R. Curto, Fredholm and invertible tuples of operators, the deformation problem, Trans. Amer. Math. Soc. 226 (1981), 129-159.

3. _ Reinhardt domains and operator theory, Proc. Sympos. Pure Math., vol. 52, part 3, Amer. Math. Soc., Providence, R. I., 1990, pp. 93-101.

4. R. Curto and P. Muhly, $C^{*}$-algebras of multiplication operators on Bergman spaces, $\mathbf{J}$. Funct. Anal. 61 (1985), 315-329.

5. R. Curto and N. Salinas, Spectral properties of cyclic subnormal m-tuples, Amer. J. Math. 107 (1985), 113-138. 
6. J. Dixmier, $C^{*}$-algebras and their representations, North-Holland, Amsterdam, 1977.

7. L. Hormander, Introduction to complex analysis in several variables, North-Holland, Amsterdam, 1973.

8. __ The analysis of linear partial differential operators, Springer-Verlag, New York, 1983.

9. S. Krantz, Function theory of several complex variables, Wiley, New York, 1982.

10. Q. Lin and N. Salinas, Proper holomorphic map and analytic Toeplitz $N$-tuples, Indiana Univ. Math. J. 39 (1990), 547-562.

11. P. Muhly and J. Renault, $C^{*}$-algebras of multivariable Wiener-Hopf operators, Trans. Amer. Math. Soc. 274 (1982), 1-44.

12. A. Nica, Some remarks on the groupoid approach to Wiener-Hopf operators, J. Operator Theory 18 (1987), 163-198.

13. E. Park, Index theory and Toeplitz algebras on certain cones in $\mathbf{Z}^{2}$, J. Operator Theory 23 (1990), 125-146.

14. G. Pedersen, $C^{*}$-algebras and their automorphism groups, London Math. Soc. Monographs, vol. 10, Academic Press, London, 1979.

15. M. Range, Holomorphic functions and integral representation in several complex variables, Springer-Verlag, Berlin and New York, 1986.

16. J. Renault, A groupoid approach to $C^{*}$-algebras, Lecture Notes in Math., vol. 793, SpringerVerlag, New York, 1980.

17. N. Salinas, The $\bar{\partial}$-formalism and the $C^{*}$-algebra of the Bergman $n$-tuple, J. Operator Theory 22 (1989), 325-343.

18. _ Non-compactness of the $\bar{\partial}$-Neumann problem and Toeplitz $C^{*}$-algebras, Proc. Sympos. Pure Math., vol. 52, part 3, Amer. Math. Soc., Providence, R. I., 1991, pp. 329-334.

19. N. Salinas, A. Sheu, and H. Upmeier, Toeplitz operators on pseudoconvex domains and foliation $C^{*}$-algebras, Ann. of Math. 130 (1989), 531-565.

20. N. Salinas and H. Upmeier, Holomorphic foliations and Toeplitz $C^{*}$-algebras (in preparation).

21. A. G. Sergeev, On matrix Reinhardt domains, preprint, 1989.

22. A. Sheu, On the isomorphism between Toeplitz $C^{*}$-algebras of the Hardy and the Bergman spaces, Proc. Amer. Math. Soc. 116 (1992), 113-120.

23. A. Shields, Weighted shift operators and analytic function theory, Math. Surveys Monographs, vol. 13, Amer. Math. Soc., Providence, R. I., 1974, pp. 49-128.

Department of Mathematics, University of Kansas at Lawrence, Lawrence, Kansas $66045-2142$ 